\title{
Farthest Neighbor: The Distant Milky Way Satellite Eridanus II ${ }^{*}$
}

\author{
T. S. Li ${ }^{1,2}$, J. D. Simon ${ }^{3}$, A. Drlica-Wagner ${ }^{1}$, K. Bechtol ${ }^{4,5,6}$, M. Y. Wang ${ }^{2}$, J. García-Bellido ${ }^{7}$, J. Frieman ${ }^{1,8}$, J. L. Marshall ${ }^{2}$,
} D. J. James ${ }^{9,10}$, L. Strigari ${ }^{2}$, A. B. Pace ${ }^{2}$, E. Balbinot ${ }^{11}$, Y. Zhang ${ }^{1}$, T. M. C. Abbott ${ }^{10}$, S. Allam ${ }^{1}$, A. Benoit-Lévy ${ }^{12,13,14}$, G. M. Bernstein ${ }^{15}$, E. Bertin ${ }^{12,14}$, D. Brooks ${ }^{13}$, D. L. Burke ${ }^{16,17}$, A. Carnero Rosell ${ }^{18,19}$, M. Carrasco Kind ${ }^{20,21}$, J. Carretero ${ }^{22,23}$, C. E. Cunha ${ }^{16}$, C. B. D'Andrea ${ }^{24,25}$, L. N. da Costa $^{18,19}$, D. L. DePoy ${ }^{2}$, S. Desai ${ }^{26}$, H. T. Diehl ${ }^{1}$, T. F. Eifler ${ }^{27}$, B. Flaugher ${ }^{1}$, D. A. Goldstein ${ }^{28,29}$, D. Gruen ${ }^{16,17}$, R. A. Gruendl ${ }^{20,21}$, J. Gschwend ${ }^{18,19}$, G. Gutierrez ${ }^{1}$, E. Krause ${ }^{16}$, K. Kuehn ${ }^{30}$, H. Lin $^{1}$,

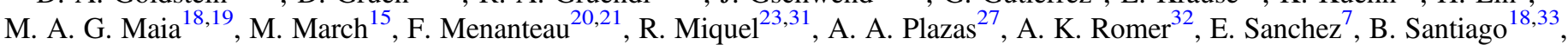
M. Schubnell ${ }^{34}$, I. Sevilla-Noarbe ${ }^{7}$, R. C. Smith ${ }^{10}$, F. Sobreira ${ }^{18,35}$, E. Suchyta ${ }^{36}$, G. Tarle ${ }^{34}$, D. Thomas ${ }^{24}$, D. L. Tucker ${ }^{1}$, A. R. Walker ${ }^{10}$, R. H. Wechsler ${ }^{16,17,37}$, W. Wester ${ }^{1}$, and B. Yanny ${ }^{1}$

(DES Collaboration)

${ }^{1}$ Fermi National Accelerator Laboratory, P. O. Box 500, Batavia, IL 60510, USA
${ }^{2}$ George P. and Cynthia Woods Mitchell Institute for Fundamental Physics and Astronomy, and Department of Physics and Astronomy, Texas A\&M University, College Station, TX 77843, USA

${ }^{3}$ Observatories of the Carnegie Institution of Washington, 813 Santa Barbara Street, Pasadena, CA 91101, USA

${ }^{4}$ LSST, 933 North Cherry Avenue, Tucson, AZ 85721, USA

${ }^{5}$ Wisconsin IceCube Particle Astrophysics Center (WIPAC), Madison, WI 53703, USA

${ }^{6}$ Department of Physics, University of Wisconsin-Madison, Madison, WI 53706, USA

${ }^{7}$ Instituto de Física Teórica UAM/CSIC, Universidad Autónoma de Madrid, Cantoblanco, E-28049 Madrid, Spain

${ }_{9}^{8}$ Kavli Institute for Cosmological Physics, University of Chicago, Chicago, IL 60637, USA

${ }^{9}$ Astronomy Department, University of Washington, Box 351580, Seattle, WA 98195 , USA

${ }^{10}$ Cerro Tololo Inter-American Observatory, National Optical Astronomy Observatory, Casilla 603, La Serena, Chile

${ }^{11}$ Department of Physics, University of Surrey, Guildford GU2 7XH, UK

${ }^{12}$ CNRS, UMR 7095, Institut d'Astrophysique de Paris, F-75014, Paris, France

${ }^{13}$ Department of Physics \& Astronomy, University College London, Gower Street, London, WC1E 6BT, UK

${ }^{14}$ Sorbonne Universités, UPMC Univ Paris 06, UMR 7095, Institut d'Astrophysique de Paris, F-75014, Paris, France

${ }^{15}$ Department of Physics and Astronomy, University of Pennsylvania, Philadelphia, PA 19104, USA

${ }^{16}$ Kavli Institute for Particle Astrophysics \& Cosmology, P. O. Box 2450, Stanford University, Stanford, CA 94305, USA

${ }^{17}$ SLAC National Accelerator Laboratory, Menlo Park, CA 94025, USA

${ }^{18}$ Laboratório Interinstitucional de e-Astronomia-LIneA, Rua Gal. José Cristino 77, Rio de Janeiro, RJ-20921-400, Brazil

${ }^{19}$ Observatório Nacional, Rua Gal. José Cristino 77, Rio de Janeiro, RJ-20921-400, Brazil

${ }^{20}$ Department of Astronomy, University of Illinois, 1002 West Green Street, Urbana, IL 61801, USA

${ }^{21}$ National Center for Supercomputing Applications, 1205 West Clark Street, Urbana, IL 61801, USA

${ }^{22}$ Institut de Ciències de l'Espai, IEEC-CSIC, Campus UAB, Carrer de Can Magrans, s/n, E-08193 Bellaterra, Barcelona, Spain

${ }^{23}$ Institut de Física d'Altes Energies (IFAE), The Barcelona Institute of Science and Technology, Campus UAB, E-08193 Bellaterra (Barcelona) Spain

${ }_{25}^{24}$ Institute of Cosmology \& Gravitation, University of Portsmouth, Portsmouth, PO1 3FX, UK

${ }^{25}$ School of Physics and Astronomy, University of Southampton, Southampton, SO17 1BJ, UK

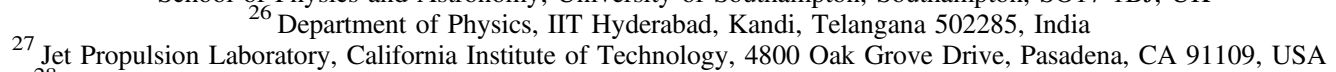

${ }^{28}$ Department of Astronomy, University of California, Berkeley, 501 Campbell Hall, Berkeley, CA 94720, USA

${ }^{29}$ Lawrence Berkeley National Laboratory, 1 Cyclotron Road, Berkeley, CA 94720, USA

${ }^{30}$ Australian Astronomical Observatory, North Ryde, NSW 2113, Australia

${ }^{31}$ Institució Catalana de Recerca i Estudis Avançats, E-08010 Barcelona, Spain

${ }^{32}$ Department of Physics and Astronomy, Pevensey Building, University of Sussex, Brighton, BN1 9QH, UK

${ }^{33}$ Instituto de Física, UFRGS, Caixa Postal 15051, Porto Alegre, RS-91501-970, Brazil

${ }^{34}$ Department of Physics, University of Michigan, Ann Arbor, MI 48109, USA

${ }^{35}$ Universidade Federal do ABC, Centro de Ciências Naturais e Humanas, Avenida dos Estados, 5001, Santo André, SP, 09210-580, Brazil

${ }^{36}$ Computer Science and Mathematics Division, Oak Ridge National Laboratory, Oak Ridge, TN 37831, USA

Department of Physics, Stanford University, 382 Via Pueblo Mall, Stanford, CA 94305, USA

Received 2016 November 14; revised 2017 February 8; accepted 2017 February 14; published 2017 March 17

\begin{abstract}
We present Magellan/IMACS spectroscopy of the recently discovered Milky Way satellite Eridanus II (Eri II). We identify 28 member stars in Eri II, from which we measure a systemic radial velocity of $v_{\text {hel }}=75.6 \pm 1.3$ (stat.) \pm 2.0 (sys.) $\mathrm{km} \mathrm{s}^{-1}$ and a velocity dispersion of $6.9_{-0.9}^{+1.2} \mathrm{~km} \mathrm{~s}^{-1}$. Assuming that Eri II is a dispersion-supported system in dynamical equilibrium, we derive a mass within the half-light radius of $1.2_{-0.3}^{+0.4} \times 10^{7} M_{\odot}$, indicating a mass-tolight ratio of $420_{-140}^{+210} M_{\odot} / L_{\odot}$ and confirming that it is a dark matter-dominated dwarf galaxy. From the equivalent width measurements of the $\mathrm{Ca}$ triplet lines of 16 red giant member stars, we derive a mean metallicity of [Fe/ $\mathrm{H}]=-2.38 \pm 0.13$ and a metallicity dispersion of $\sigma_{[\mathrm{Fe} / \mathrm{H}]}=0.47_{-0.09}^{+0.12}$. The velocity of Eri II in the Galactic standard of rest frame is $v_{\mathrm{GSR}}=-66.6 \mathrm{~km} \mathrm{~s}^{-1}$, indicating that either Eri II is falling into the Milky Way potential for the first time or that it has passed the apocenter of its orbit on a subsequent passage. At a Galactocentric distance of $\sim 370 \mathrm{kpc}$, Eri II is one of the Milky Way's most distant satellites known. Additionally, we show that
\end{abstract}

\footnotetext{
* This paper includes data gathered with the 6.5 meter Magellan Telescopes

located at Las Campanas Observatory, Chile.
} 
the bright blue stars previously suggested to be a young stellar population are not associated with Eri II. The lack of gas and recent star formation in Eri II is surprising given its mass and distance from the Milky Way, and may place constraints on models of quenching in dwarf galaxies and on the distribution of hot gas in the Milky Way halo. Furthermore, the large velocity dispersion of Eri II can be combined with the existence of a central star cluster to constrain massive compact halo object dark matter with mass $\gtrsim 10 M_{\odot}$.

Key words: dark matter - galaxies: dwarf - galaxies: individual (Eridanus II) - galaxies: stellar content Local Group - stars: abundances

\section{Introduction}

Over the past two years, more than 20 ultra-faint dwarf galaxy candidates have been discovered in data from the Dark Energy Survey (DES; Bechtol et al. 2015; Drlica-Wagner et al. 2015b; Kim \& Jerjen 2015; Koposov et al. 2015) and other large optical surveys (Kim et al. 2015a, 2015b; Laevens et al. 2015b, 2015a; Martin et al. 2015; Drlica-Wagner et al. 2016; Homma et al. 2016; Torrealba et al. 2016a, 2016b). One of the largest most luminous and most distant newly discovered satellites is Eridanus II (Eri II), which has an absolute magnitude of $M_{V} \sim-7$, a half-light radius of $r_{h} \sim 280 \mathrm{pc}$, and a Galactocentric distance of $D \sim 370 \mathrm{kpc}$ (Bechtol et al. 2015; Koposov et al. 2015; Crnojević et al. 2016; see Table 1).

Eri II is most likely located just beyond the virial radius of the Milky Way, which is typically estimated to be $\sim 300 \mathrm{kpc}$ (Bland-Hawthorn \& Gerhard 2016; Taylor et al. 2016). This places Eri II in a sharp transition region between the gas-free dwarf spheroidals (with $D \lesssim 250 \mathrm{kpc}$ ) and the more distant gas-rich star-forming dwarfs (Einasto et al. 1974; Blitz \& Robishaw 2000; Grcevich \& Putman 2009; Spekkens et al. 2014). Koposov et al. (2015) suggested that Eri II may contain a young stellar population component ( 250 Myr) because a few candidate blue-loop stars are spatially coincident, and therefore Eri II would be similar to the gas-rich dwarf Leo T, which is slightly more distant and more luminous $\left(D=420 \mathrm{kpc}, M_{V} \sim-8\right.$; Irwin et al. 2007; de Jong et al. 2008; Ryan-Weber et al. 2008) and has undergone multiple epochs of star formation (de Jong et al. 2008; Weisz et al. 2012). If this were the case, Eri II would be the least luminous star-forming galaxy known. However, Westmeier et al. (2015) measured the H I gas content using HIPASS data (Barnes et al. 2001) and did not detect any $\mathrm{H}$ I gas associated with Eri II. Crnojević et al. (2016) also obtained H I observations from the Green Bank Telescope and found a more stringent upper limit on the $\mathrm{HI}$ mass $\left(M_{\mathrm{HI}}<2800 M_{\odot}\right)$, indicating that Eri II is an extremely gas-poor system. With deep imaging from Magellan/Megacam, Crnojević et al. (2016) found a possible intermediate-age $(\sim 3 \mathrm{Gyr})$ population in Eri II. Moreover, they confirmed that there is a star cluster whose projected position is very close to the center of Eri II, making it the least luminous galaxy known to host a (possibly central) star cluster.

The features described above make Eri II one of the most interesting of the newly discovered Milky Way satellites for spectroscopic study. In this paper we present the first spectroscopic observations of Eri II, from which we determine its dark matter content and test for the existence of a young stellar population. In Section 2 we describe the observations, target selection, and data reduction. In Section 3 we perform velocity and metallicity measurements on the observed stars in Eri II and identify spectroscopic members. We then compute the global properties of Eri II and discuss its nature and origin in Section 4, and we conclude in Section 5.

\section{Observations and Data Reduction}

\subsection{Observations and Target Selection}

We observed Eri II with the IMACS spectrograph (Dressler et al. 2006) on the Magellan Baade telescope on the nights of 2015 October 16-17 and 2015 November 18-19. The observing conditions on both runs were clear, with seeing that varied from 0 " 6 to $0 . " 9$. The observing procedure and instrument setup are similar to the observation of the Tucana III (Tuc III) dwarf galaxy described by Simon et al. (2016). For both runs we used the $\mathrm{f} / 4$ camera on IMACS, which has an $8192 \times 8192$ mosaic provided by a $4 \times 2$ array of $2048 \times 4096$ pixel e2v CCDs. The spectrograph was configured with the $1200 \mathrm{\ell} / \mathrm{mm}$ grating blazed at 32.7. This setup produces a spectral dispersion of $0.19 \AA^{\circ} \mathrm{pix}^{-1}$, a peak throughput above $14 \%$ for $7800-8800 \AA$, and a spectral resolution of $R \sim 11,000$ for a 0 ". 7 slit width. We used a tilt angle of $32^{\circ} .4$ to provide a minimum wavelength range of $7550-8750 \AA$ for each slit, with typical wavelength coverage of 7400-9000 А. The WB5600-9200 filter was used to block shorter wavelength light. This wavelength range covers the $\mathrm{Ca}$ triplet (CaT) absorption lines around $8500 \AA$, which are used for measuring radial velocities and metallicities of candidate member stars, as well as the telluric absorption lines (Fraunhofer A-band) around $7600 \AA$, which are used for the corrections of velocity errors caused by miscentering of the stars within the slits (see Section 3.1 and Sohn et al. 2007 for details). While the $f / 4$ camera on IMACS provides a full field of view of $15 ! 4 \times 15 ! 4$ for multislit spectroscopy, we limited the placement of slits to a 15 ! $4 \times 8^{\prime}$ portion to ensure that all the spectra span the required wavelength range for accurate velocity measurements.

We observed the candidate member stars in Eri II with one slitmask. The spectroscopic targets were selected using photometry from the coadded images of the first internal annual release of DES data (Y1A1; Bechtol et al. 2015). Since Eri II is a distant Milky Way satellite, most of the candidate stars brighter than $g=23$ are near the tip of the red giant branch (RGB). We chose spectroscopic targets using a PARSEC isochrone (Bressan et al. 2012) with age $=12 \mathrm{Gyr}$ and $[\mathrm{Fe} / \mathrm{H}]=-2.2$ as guidance. RGB candidates were selected as stars within $0.13 \mathrm{mag}$ of the isochrone, brighter than $g=22.5$, and within $7^{\prime}$ (three times the half-light radius) of the center of Eri II. In addition, we targeted potential blueloop stars within a box defined by $0.2<g-r<0.4$ and $20.3<g<20.6$. The relative priorities for RGB stars were based on brightness and projected distance from the center of the galaxy, and for blue-loop candidates the priorities were based only on projected distance on the sky (since all of the stars have similar magnitudes). The remaining mask space was filled with stars that have photometry that makes them unlikely to be members. This selection process resulted in the placement of 680 !" $7 \times 5$ ! 0 slitlets on the slitmask. We observed this mask for a total of $3 \mathrm{hr}$ in the October run and $9 \mathrm{hr}$ in the November run. To ensure accurate velocity measurements, 


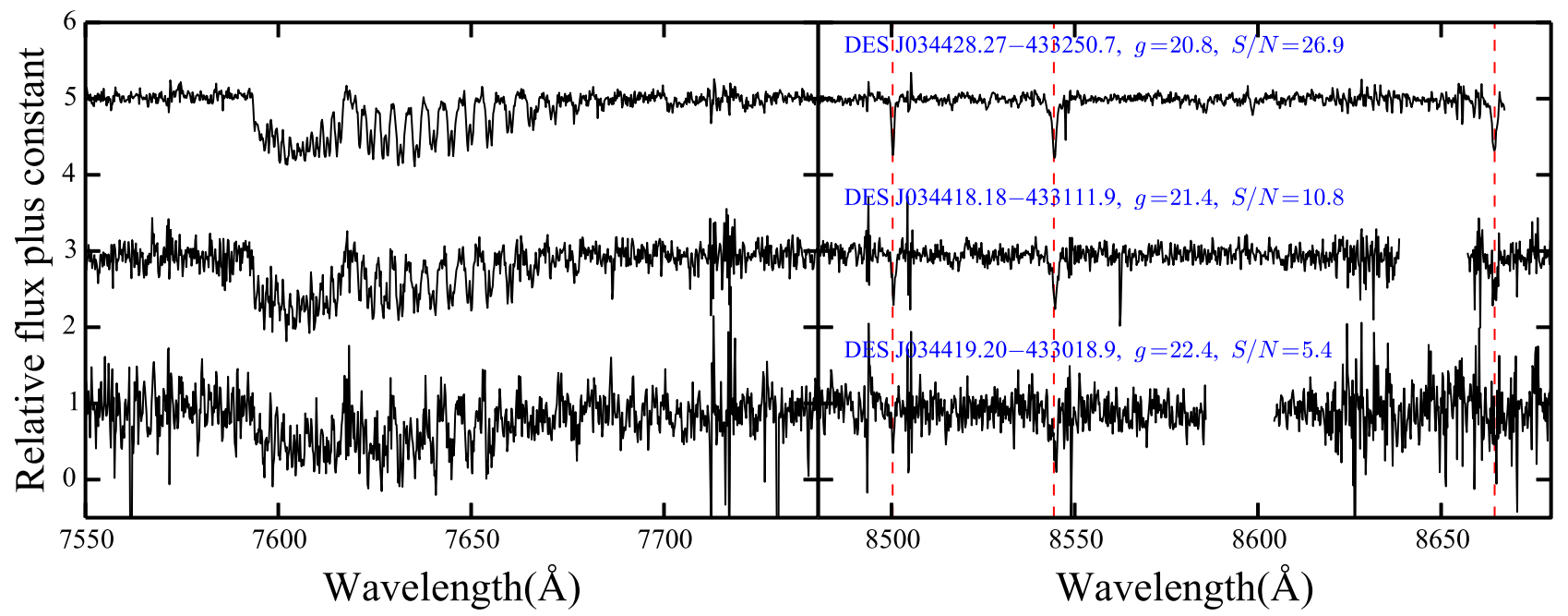

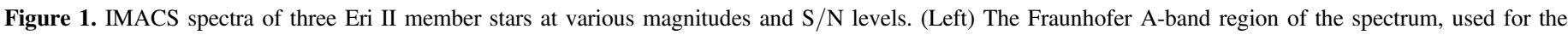

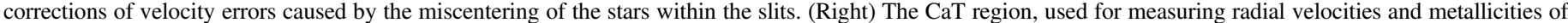
candidate member stars. The three Ca lines are marked with dashed red lines. The gaps in the spectra are caused by the gaps between IMACS CCDs.

after every two 30-40 minutes science exposures, we acquired one wavelength calibration frame and one flatfield frame at the same position as the science exposure. For the October run, we used $\mathrm{He}, \mathrm{Ne}$, and $\mathrm{Ar}$ comparison lamps for wavelength calibration, while for the November run, we used $\mathrm{Kr}$, Ne, and $\mathrm{Ar}$ lamps. The $\mathrm{Kr}$ lamp provides additional strong lines in the critical 7600-7900 $\AA$ wavelength range where there are only few usable Ne and Ar lines; this improved the wavelength calibration around the Fraunhofer A-band.

In addition to the observations targeting candidate members of Eri II, we also obtained spectra of several metal-poor stars to serve as radial velocity templates for the velocity measurements, and a hot rapid rotator (HR 4781) to serve as a telluric template for the velocity error corrections. More templates were also obtained during additional IMACS runs with identical observing setups. For both the radial velocity templates and the telluric template, we obtained the spectra using a north-south oriented longslit while driving the stars perpendicularly across the slit (i.e., across the 0.7 dimension) at a constant rate during the exposure. These spectra simulate a source that uniformly fills the slit, and thus they accurately reflect the mean integrated slit function.

\subsection{Data Reduction}

We reduced the IMACS spectra following the procedures described by Simon et al. (2016) for Tuc III. The reduction procedures include bias subtraction, removal of read-out pattern noise, an initial wavelength solution and slit mapping with the Cosmos pipeline (Dressler et al. 2011), and a refined wavelength calibration and spectral extraction using an IMACS pipeline derived from the DEEP2 data reduction pipeline for Keck/DEIMOS (Cooper et al. 2012).

Each individual science frame was reduced using the corresponding flatfield frame and wavelength calibration frame. The end products of the pipeline are the extracted 1D spectra and the corresponding inverse-variance spectra. Of the targeted 68 stars, 66 were successfully extracted, and 2 stars fell onto chip gaps or off the detector array. We then combined the extracted spectra from each observing run using inversevariance weighting. As the November run has a much longer total exposure time and better seeing, we used the coadded spectra from the November run for the kinematic measurements in later sections. We kept the coadded spectra from the October run separate from the coadded spectra from the November run to test the possible radial velocity variation from binary orbital motion. For the November coadded spectra, we reached a signal-to-noise ratio $(\mathrm{S} / \mathrm{N})$ of $\sim 5$ per pixel for stars at $g \sim 22$ and $\mathrm{S} / \mathrm{N} \sim 30$ per pixel for stars at $g \sim 20.5$. For the October coadded spectra, the $\mathrm{S} / \mathrm{N}$ is about a factor of two lower. Finally, all the coadded spectra were normalized to unity in the continuum by fitting a second-order polynomial. Examples of the spectra at various brightness and $\mathrm{S} / \mathrm{N}$ levels are shown in Figure 1.

We reduced the spectra for the velocity and telluric templates in the same manner as the science exposures described above. For the telluric template, we set the regions outside of telluric absorption bands to unity; for the velocity templates, we set the regions inside the telluric bands to unity and shifted them to rest frame.

\section{Velocity and Metallicity Measurements \\ 3.1. Radial Velocity Measurements}

We measured radial velocities by fitting reduced spectra with velocity templates using a Markov chain Monte Carlo (MCMC) sampler (emcee; Foreman-Mackey et al. 2013) ${ }^{38}$ and a likelihood function defined as

$$
\log \mathcal{L}=-\frac{1}{2} \sum_{\lambda=\lambda_{1}}^{\lambda_{2}} \frac{\left[f_{s}(\lambda)-f_{\text {std }}\left(\lambda\left(1+\frac{v}{c}\right)\right)\right]^{2}}{\sigma_{s}^{2}(\lambda)} .
$$

We note that the log-likelihood is defined up to an additive constant. Here, $f_{s}(\lambda)$ and $\sigma_{s}^{2}(\lambda)$ are the normalized spectrum and its corresponding variance, and $f_{\text {std }}(\lambda)$ is the normalized velocity template. For the velocity measurement, we primarily used the CaT feature, and therefore we set $\lambda_{1}=8450 \AA$ and $\lambda_{2}=8700 \AA$. Our procedure fits the radial velocity by shifting the velocity template by a velocity $v$ to maximize the likelihood

\footnotetext{
$\overline{38 \text { emcee v2.2.0: http://dan.iel.fm/emcee/. }}$.
} 

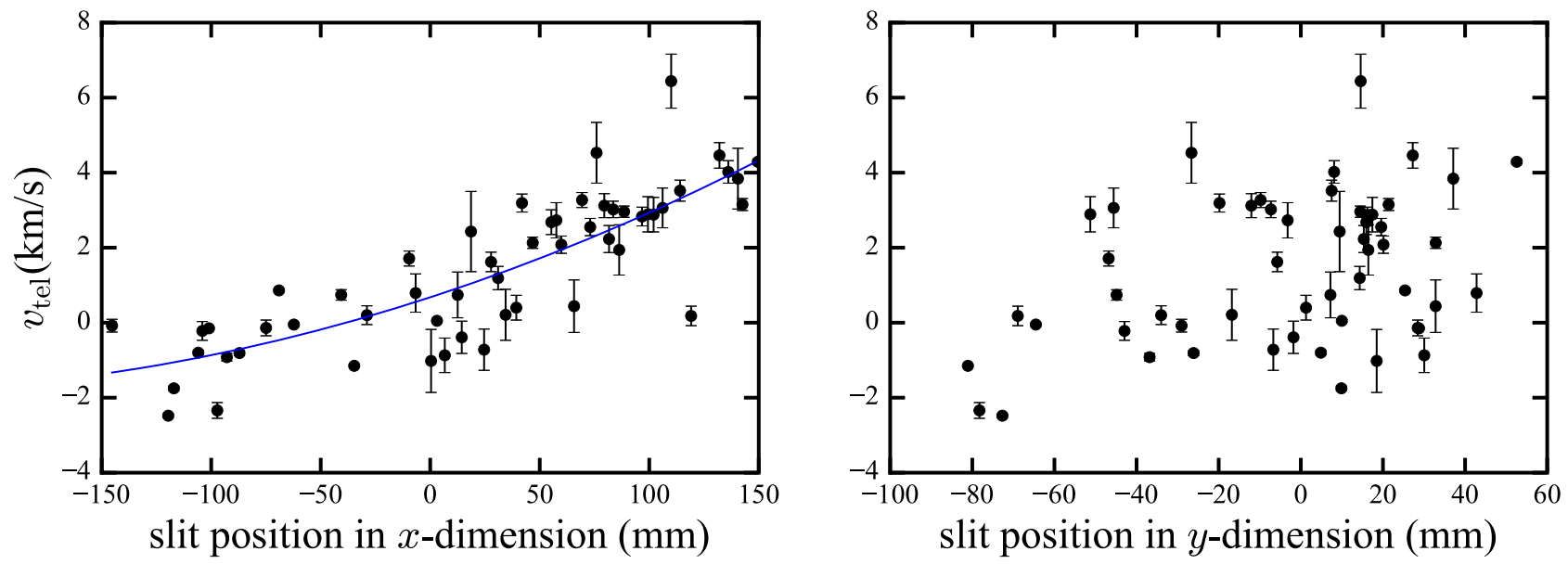

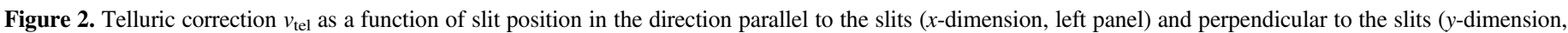
right panel). The rms of $v_{\text {tel }}$ residuals in the $x$-dimension with respect to a fitted second-order polynomial is $\sim 1 \mathrm{~km} \mathrm{~s}^{-1}$.

function. For this paper, we use the metal-poor RGB star HD 122563 as the template for all of the science spectra.

For each spectrum, we ran an MCMC sampler with 20 walkers that each made 1000 steps including a burn-in stage of 50 steps. We used the median and the standard deviation (with $5 \sigma$ clipping) of the posterior distributions as the measured velocity $v_{\mathrm{obs}}$ and velocity error $\sigma_{v_{\mathrm{obs}}}$ for each star.

We then determined and applied a telluric correction to each velocity measurement to account for velocity errors that result from miscentering the star within the slit. We ran the same MCMC sampler as described above, but instead used a telluric template and a fitting wavelength range of 7550-7700 А. For each spectrum, the telluric correction $v_{\text {tel }}$ and uncertainty $\sigma_{v_{\text {tel }}}$ were obtained from the posterior distribution from the MCMC sampler.

In Figure 2 we show the telluric correction $v_{\text {tel }}$ as a function of slit position in the direction parallel to the slits $(x)$ and perpendicular to the slits $(y)$. The correction generally ranges between -2 and $4 \mathrm{~km} \mathrm{~s}^{-1}$ for this mask and shows a dependence on the slit position in the spatial direction. The rms of the residuals after a second-order polynomial fit to the data is $\sim 1 \mathrm{~km} \mathrm{~s}^{-1}$. The systematic trend (i.e., polynomial fit) of this telluric correction is most likely caused by either a small mask rotation or anamorphic demagnification of the IMACS spectrograph; the scatter around the fit (i.e., rms) may be associated with the astrometric errors of DES Y1A1 data and the systematic uncertainty in the velocity correction determination.

The velocity was then calculated as $v=v_{\mathrm{obs}}-v_{\text {tel }}$ and the statistical uncertainty of $v$ was calculated as $\sigma_{v_{\text {stat }}}=\sqrt{\sigma_{v_{\text {obs }}}^{2}+\sigma_{v_{\text {tel }}}^{2}}$. We note that we used $v_{\text {tel }}$ from individual stars for the telluric correction rather than the polynomial fit shown in Figure 2. It is worth noting that $\sigma_{v_{\text {stat }}}$ is only the statistical uncertainty on the velocity measurements, which is associated with the $S / N$ of the spectra. For high $\mathrm{S} / \mathrm{N}$ spectra, the velocity measurement can be very precise. However, the accuracy of the velocity measurement is limited by systematic effects, such as instrument flexure, uncertainties in the wavelength calibration, uncertainties in the template velocity, template mismatching, and the uncertainties in the telluric correction. These systematic uncertainties should also be considered in the total error budget. We estimated the systematic uncertainty as the quadrature difference between repeat measurements and the statistical uncertainty (cf. Simon \& Geha 2007; Simon et al. 2015). Similar to Simon et al. (2016), we found that this systematic uncertainty is $\sigma_{v_{\mathrm{sys}}}=1.2 \mathrm{~km} \mathrm{~s}^{-1}$ for the October observations and $\sigma_{v_{\text {sys }}}=1.0 \mathrm{~km} \mathrm{~s}^{-1}$ for the November observations. The slight difference in the systematic errors between the two observing runs is mainly caused by the new $\mathrm{Kr}$ lamp that was included in November: it improved the wavelength solution at the blue end. We added this systematic uncertainty in quadrature with the statistical uncertainties as the final reported velocity uncertainties, $\sigma_{v}=\sqrt{\sigma_{v_{\text {stat }}}^{2}+\sigma_{v_{\mathrm{sys}}}^{2}}$.

Of the 66 extracted spectra, 54 have high enough $\mathrm{S} / \mathrm{N}$ to determine velocities and velocity uncertainties using the aforementioned method. Finally, all velocity measurements are transformed into the heliocentric frame. The results are listed in Table 2.

In order to confirm that our error estimation is reasonable, we selected 38 stars that have measured velocities and velocity uncertainties from both the November run and October run and computed the distribution of velocity differences between the two independent measurements $\left(v_{1}, v_{2}\right)$, divided by the quadrature sum of their uncertainties $\left(\sqrt{\sigma_{1}^{2}+\sigma_{2}^{2}}\right) ; v_{1}$ and $\sigma_{1}$ are the measurements from October and $v_{2}$ and $\sigma_{2}$ are the measurements from November. The resulting distribution shown in Figure 3 is well described by a normal distribution with zero mean and unit variance, shown as the red dashed curve in the same plot. A Kolmogorov-Smirnov (K-S) test of the repeated measurements against the normal distribution gives a $p$-value of 0.98 , confirming that our error model provides an accurate description of the velocity uncertainties. Since we do not see any outliers when comparing the results between the two observing runs, we conclude that we are not able to detect any binary stars in Eri II based on the one-month baseline. Binaries with longer periods might be present, but detecting them would require additional observations with a longer time baseline.

\subsection{Spectroscopic Membership Determination}

The color-magnitude diagram (CMD), spatial distribution, and velocity distribution of the candidate stars are displayed in Figure 4. From the 54 stars with measured velocities, we found 28 Eri II members that form a narrow velocity peak at 

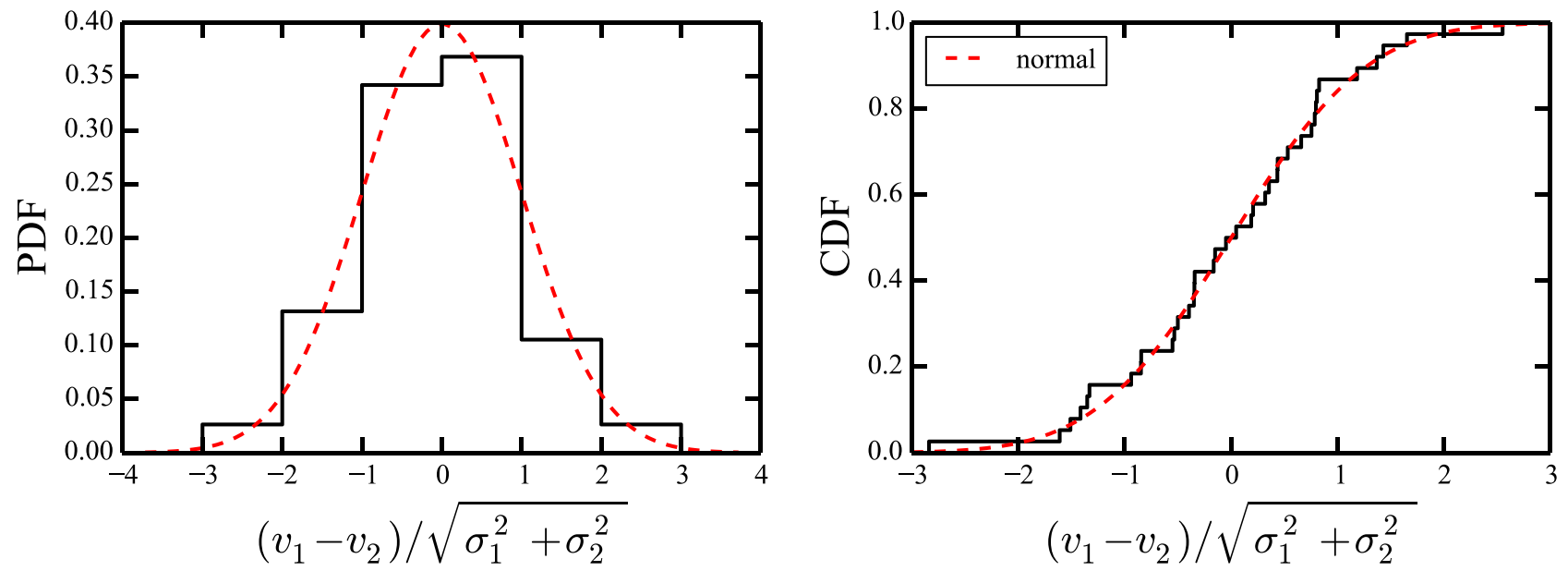

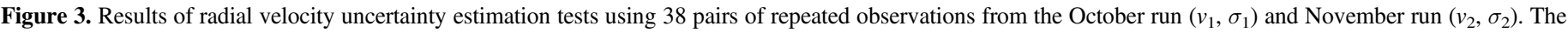

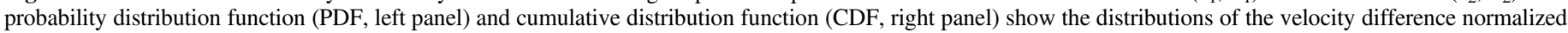

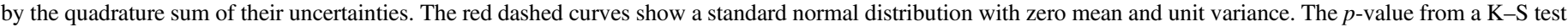
between the sample and the model is 0.98 . This indicates that our estimation of the velocity uncertainties is reasonable.

$\sim 75 \mathrm{~km} \mathrm{~s}^{-1}$ (right panel of Figure 4). For the large majority of the observed stars, membership status is unambiguous. The member stars located close to the center of Eri II and located along the isochrone in the CMD are highlighted in red in the histogram and denoted as red filled circles. Stars with $v_{\text {hel }}>140 \mathrm{~km} \mathrm{~s}^{-1}$ or $v_{\text {hel }}<30 \mathrm{~km} \mathrm{~s}^{-1}$ that are clearly not associated with Eri II are shown by gray filled circles in the left and middle panels of Figure 4. Several candidate members have a velocity close to the peak and are highlighted in cyan in the histogram, and they are also denoted as cyan filled circles in the left and middle panels of Figure 4. These stars are classified as non-members since they are located far from the RGB isochrone in the CMD. While DES J034404.78-432727.7 lies close to the RGB isochrone, it is more than two half-light radii away from the center of Eri II along the minor axis and has strong $\mathrm{Na}$ I lines. In fact, all the stars coded in cyan show strong Na I lines at $\lambda=8183 \AA$ and $\lambda=8195 \AA$ (see Figure 5 as an example), indicating that they are foreground M-dwarf stars rather than distant giants (Schiavon et al. 1997).

Finally, we used the Besançon (Robin et al. 2003) Galactic stellar model to estimate the expected number of foreground main-sequence stars in our spectroscopic sample. We selected simulated stars within $0.2 \mathrm{mag}$ of the PARSEC isochrone and with $20.5<g<22.5$ (i.e., the location of the red filled circles in the CMD in the left panel of Figure 4). We found $\sim 70$ simulated stars that have a velocity consistent with the heliocentric velocity peak of Eri II $\left(60-90 \mathrm{~km} \mathrm{~s}^{-1}\right)$ in an area of $1 \mathrm{deg}^{2}$ centered on Eri II. When scaled to the area within two half-light radii of Eri II $\left(\sim 0.02 \mathrm{deg}^{2}\right)$, the foreground contamination is expected to be $1-2$ stars.

\subsection{Metallicity Measurements}

We measured the metallicity of the red giant members using the equivalent widths (EWs) of the CaT lines. Following the procedure described by Simon et al. (2015) and Simon et al. (2016), we fit all three of the CaT lines with a Gaussian plus Lorentzian function and then converted the summed EWs of the three $\mathrm{CaT}$ lines into metallicity using the calibration relation from Carrera et al. (2013). Because the horizontal branch stars of Eri II are too faint for accurate measurements in DES imaging, we used the absolute $V$ magnitude for the CaT calibration. We first performed the color-transformation from DES- $g$ and DES- $r$ to apparent $V$ magnitude using Equation (5) in Bechtol et al. (2015) and then adopted the distance modulus $(m-M)=22.8$ derived by Crnojević et al. (2016) to calculate absolute magnitudes.

Of the 28 spectroscopic members determined in Section 3.2, 16 have successful metallicity measurements. For the other members either the $\mathrm{S} / \mathrm{N}$ is not high enough for $\mathrm{EW}$ measurements, or one of the three $\mathrm{Ca}$ lines falls onto the CCD chip gap. The measured metallicities are reported in Table 2.

The statistical uncertainties on the EWs are calculated from the Gaussian and Lorentzian fit. We then compute a systematic uncertainty of $0.2 \AA$ on the summed EWs derived with repeat measurements (using the same approach as for the systematic velocity uncertainty in Section 3.1). The final uncertainties on the EWs reported in Table 2 are the quadrature sum of the statistical and systematic uncertainties. The metallicity uncertainties shown in Table 2 are dominated by the uncertainties of the CaT EWs, with small contributions from the uncertainties of the distance modulus, the stellar photometry, and the uncertainties on the calibration parameters from Carrera et al. (2013).

\section{Discussion}

In this section we determine the global properties of Eri II and discuss its nature and origin. We then consider implications for the quenching of star formation in dwarf galaxies and constraints on the nature of dark matter.

\subsection{Velocity Dispersion and Mass}

With the 28 spectroscopically confirmed members, we calculated the systemic velocity and the velocity dispersion of Eri II using a two-parameter Gaussian likelihood function similar to that of Walker et al. (2006):

$$
\log \mathcal{L}=-\frac{1}{2}\left[\sum_{n=1}^{N} \log \left(\sigma_{v_{\text {hel }}}^{2}+\sigma_{v_{i}}^{2}\right)+\sum_{n=1}^{N} \frac{\left(v_{i}-v_{\text {hel }}\right)^{2}}{\sigma_{v_{i}}^{2}+\sigma_{v_{\text {hel }}}^{2}}\right],
$$

where $v_{\text {hel }}$ and $\sigma_{v_{\text {hel }}}$ are the systemic velocity and the velocity dispersion of Eri II, and $v_{i}$ and $\sigma_{v_{i}}$ are the velocities and 

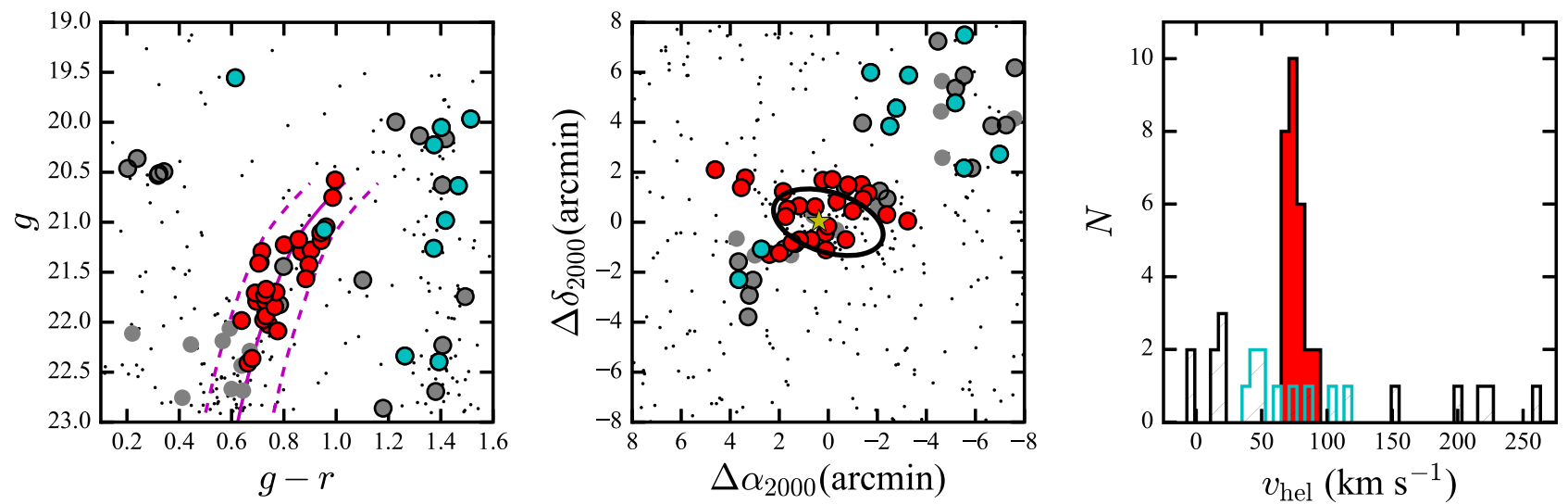

Figure 4. (Left) Color-magnitude diagram of Eri II using DES Y1A1 photometry. Stars within $8^{\prime}$ of the center of Eri II are plotted as small black dots, and stars selected for spectroscopy (as described in Section 2.1) are plotted as filled gray circles. Points surrounded by black outlines represent the stars for which we obtained successful velocity measurements. Those we identify as Eri II members are filled in with red. Non-members that have velocities close to the velocity of Eri II are filled in with cyan. A PARSEC isochrone (Bressan et al. 2012) with age $=12.0 \mathrm{Gyr}$ and $[\mathrm{Fe} / \mathrm{H}]=-2.2$ is displayed as the solid magenta line. The other two dashed magenta lines show the boundaries of the selected high-priority RGB candidates, as discussed in Section 2.1. (Middle) Spatial distribution of the observed stars. Symbols are as in the left panel. The elliptical half-light radius of Eri II is outlined as a black ellipse. The yellow star indicates the location of the central star cluster of Eri II. (Right) Radial velocity distribution of observed stars. The clear narrow peak of stars at $v \sim 75 \mathrm{~km} \mathrm{~s}^{-1}$ highlighted in red is the signature of Eri II. The hatched histogram indicates stars that are non-members of Eri II, among which the hatched cyan histogram corresponds to the cyan filled circles in the left and middle panels.
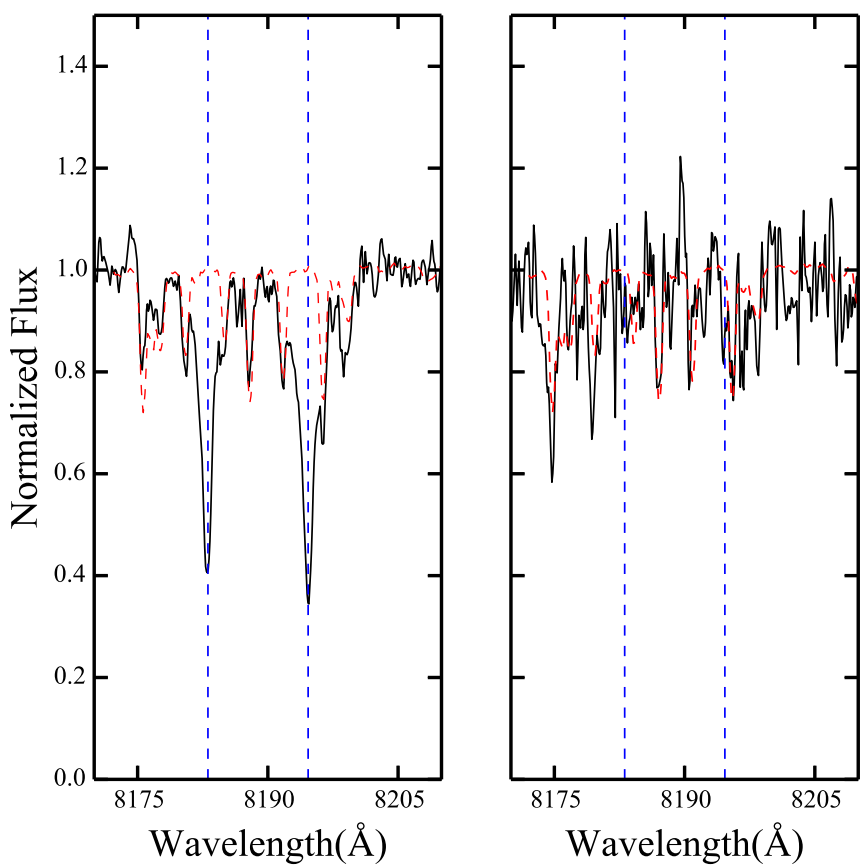

Figure 5. Example of rest frame spectra of a foreground main-sequence star (left) and an Eri II member star (right) around the Na I $\lambda 8190 \AA$ doublet, shown in black. The two Na I lines are marked with dashed blue vertical lines. The foreground dwarf has strong Na I lines, while the Eri II member is a giant star with low surface gravity and therefore the $\mathrm{Na} I$ lines are hardly detectable. Overplotted dashed red lines are the spectrum of the telluric standard star, indicating the absorption from the Earth's atmosphere.

velocity uncertainties for each member star as calculated in Section 3.1. We used an MCMC to sample the posterior distribution. We find a systemic velocity of $v_{\text {hel }}=75.6 \pm$ 1.3 (stat.) \pm 2.0 (sys.) $\mathrm{km} \mathrm{s}^{-1}$ and a velocity dispersion of $\sigma_{v_{\text {hel }}}=6.9_{-0.9}^{+1.2} \mathrm{~km} \mathrm{~s}^{-1}$, where we report the median of the posterior and the uncertainty calculated from the 16th and 84th percentiles. The systematic uncertainty $\left(2.0 \mathrm{~km} \mathrm{~s}^{-1}\right)$ on the systemic velocity is attributed to uncertainty on the velocity zeropoint of the template star. The posterior probability distribution from the MCMC sampler for the kinematic properties of Eri II is displayed on the left side of Figure 6.

In principle, the measured velocity dispersion of Eri II could be artificially inflated by the orbital motions of binary stars (McConnachie \& Côté 2010). As mentioned in Section 3.1, our observations do not span a long enough time baseline to detect any binaries. However, studies over longer time baselines have tended to show that binary stars do not have a significant impact on the velocity dispersion of classical dwarf spheroidals (Olszewski et al. 1996) or ultra-faint dwarfs (Minor et al. 2010; Simon et al. 2011). Given the large velocity dispersion of Eri II, the effect of the binaries is expected to be small, and our results should be similar even if our sample contains a few binary stars.

We calculated the mass contained within the half-light radius by adopting the mass estimator from Wolf et al. (2010), using the velocity dispersion determined above and the half-light radius of Eri II measured by Crnojević et al. (2016). The derived dynamical mass is $M_{1 / 2}=1.2_{-0.3}^{+0.4} \times 10^{7} M_{\odot}$. Given a luminosity within its half-light radius of $3.0_{-0.7}^{+0.9} \times 10^{4} L_{\odot}$, the mass-to-light ratio of Eri II is $420_{-140}^{+210} M_{\odot} / L_{\odot}$. The reported uncertainties on the dynamical mass and mass-to-light ratio include the uncertainties on the velocity dispersion, half-light radius, and luminosity.

The mass estimator from Wolf et al. (2010) is only valid for dispersion-supported stellar systems in dynamical equilibrium. Given the distance to Eri II, the system is very likely to be in dynamical equilibrium. Nevertheless, considering the high ellipticity ( $\epsilon=0.48$ ) of Eri II, we also tested the possibility of a velocity gradient, which could result either from rotational support or a tidal interaction, using a four-parameter model (i.e., mean velocity $v_{\text {hel }}$, velocity dispersion $\sigma_{v_{\text {hel }}}$, velocity gradient $\frac{d v}{d \chi}$, and position angle of the gradient $\theta$ ) similar to that 

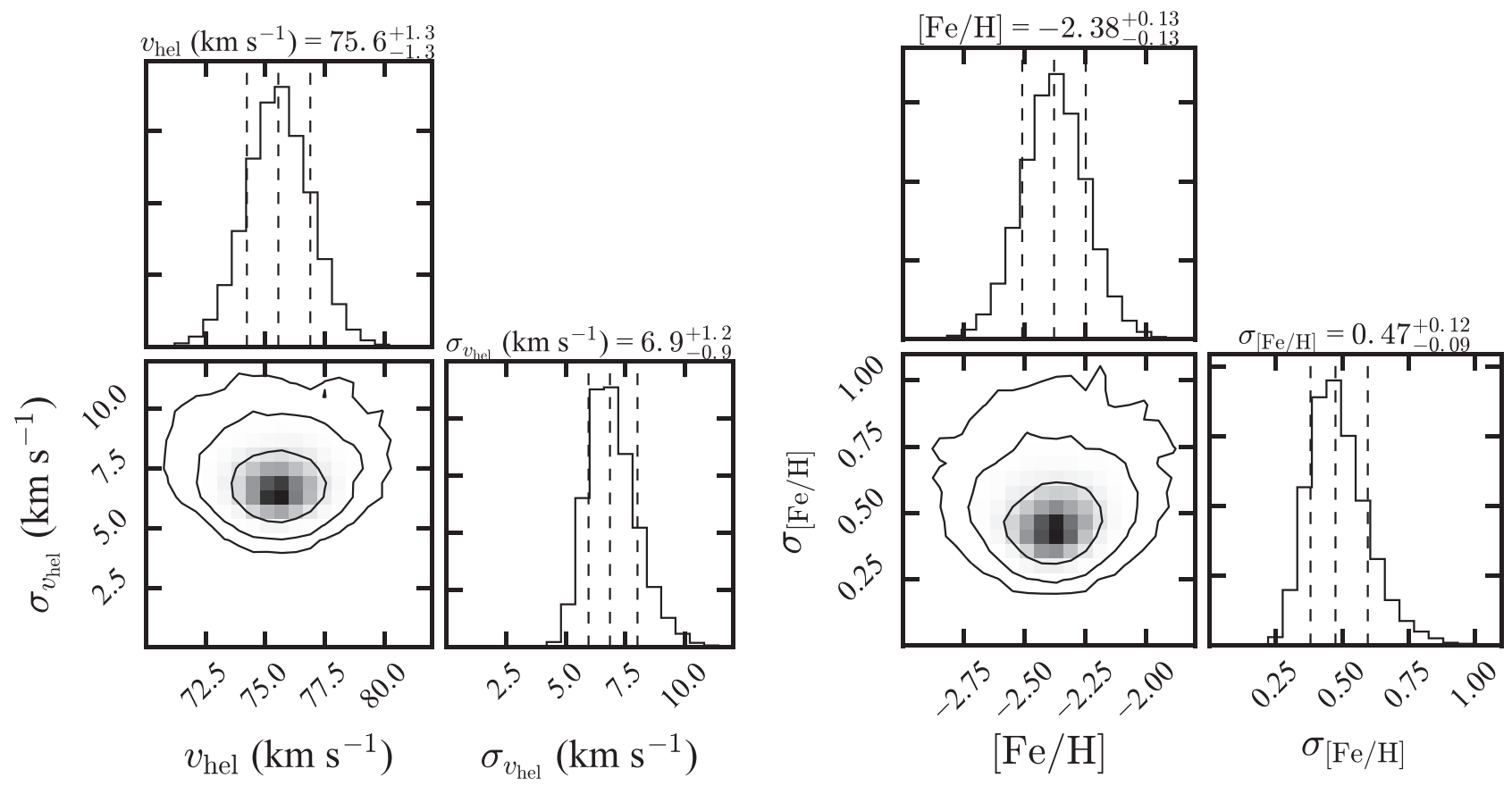

Figure 6. Two-dimensional and marginalized posterior probability distribution from an MCMC sampler using a likelihood model for the systemic velocity and velocity dispersion (left) and the mean metallicity and metallicity dispersion (right) of Eri II. For the 1D histograms, the 16th, 50th, and 84th percentiles are indicated by dashed lines. For the 2D histograms, contours represent the $68 \%, 95.5 \%$, and $99.7 \%$ confidence intervals.

of Martin \& Jin (2010) and Collins et al. (2016):

$$
\begin{aligned}
\log \mathcal{L}= & -\frac{1}{2}\left[\sum_{n=1}^{N} \log \left(\sigma_{v_{\text {hel }}}^{2}+\sigma_{v_{i}}^{2}\right)\right. \\
& \left.+\sum_{n=1}^{N} \frac{\left(v_{i}-v_{\text {hel }}-\frac{d v}{d \chi} \chi_{i}\right)^{2}}{\sigma_{v_{i}}^{2}+\sigma_{v_{\text {hel }}}^{2}}\right],
\end{aligned}
$$

where $\chi_{i}$ is the angular distance between the Eri II center $\left(\alpha_{0}\right.$, $\left.\delta_{0}\right)$ and $i$ th $\operatorname{star}\left(\alpha_{i}, \delta_{i}\right)$ projected to the gradient axis at a position angle $\theta$ :

$$
\chi_{i}=\left(\alpha_{i}-\alpha_{0}\right) \cos \left(\delta_{0}\right) \sin (\theta)+\left(\delta_{i}-\delta_{0}\right) \cos (\theta) .
$$

We note that we did not include the astrometric uncertainties of the stars in the likelihood, as the astrometric uncertainties are negligible compared to the velocity uncertainties (i.e., $\frac{d v}{d x} \sigma_{\chi_{i}} \ll \sigma_{v_{i}}$ ).

We ran a four-parameter MCMC sampler using Equations (3) and (4) to obtain the posterior probability distribution displayed in Figure 7. Since the best-fit velocity gradient, $\frac{d v}{d x}=$ $0.1 \pm 1.1 \mathrm{~km} \mathrm{~s}^{-1} \operatorname{arcmin}^{-1}$, is consistent with zero within $1 \sigma$ uncertainty, we conclude that there is no evidence of rotation or tidal interaction in Eri II, which validates the assumption used for mass derivation that Eri II is a dispersion-supported system in dynamical equilibrium.

\subsection{Metallicity Dispersion}

Using the CaT metallicity measurements, we find that the 16 brightest member stars of Eri II span more than 1 dex in iron abundance, ranging from $[\mathrm{Fe} / \mathrm{H}]=-1.7$ to $[\mathrm{Fe} / \mathrm{H}]=-3.4$. We used a Gaussian likelihood model similar to the one described in Section 4.1 to calculate the mean metallicity and metall- icity dispersion of Eri II. We find a mean metallicity of $[\mathrm{Fe} / \mathrm{H}]=-2.38 \pm 0.13$, with a dispersion of $\sigma_{[\mathrm{Fe} / \mathrm{H}]}=$ $0.47_{-0.09}^{+0.12}$. The posterior probability distributions from the MCMC sampler are displayed on the right side of Figure 6. The kinematic and chemical properties of Eri II are summarized in Table 1.

\subsection{The Classification of Eridanus II}

The mass-to-light ratio we have derived for Eri II indicates that it is a dark matter-dominated dwarf galaxy. This value is consistent with the relation between mass-to-light ratio and luminosity for other dwarf galaxies in the Milky Way and the Local Group (e.g., Simon \& Geha 2007). The low average metallicity $(-2.38 \pm 0.13)$ and large metallicity dispersion $\left(0.47_{-0.09}^{+0.12}\right)$ matches observations of other dwarf galaxies with similar luminosities (Kirby et al. 2013). Combining these results with the orbit discussed in Section 4.4 and the distance, we conclude that Eri II is one of the Milky Way's most distant satellite galaxies detected so far.

\subsection{The Orbit of Eridanus II}

The heliocentric velocity $v_{\text {hel }}=75.6 \mathrm{~km} \mathrm{~s}^{-1}$ corresponds to a velocity in the Galactic standard of rest frame ${ }^{39}$ $v_{\mathrm{GSR}}=-66.6 \mathrm{~km} \mathrm{~s}^{-1}$. Eri II is therefore moving toward us, indicating that it is either on its first infall into the Milky Way potential or that it has passed the apocenter of the orbit on a subsequent passage. To assess whether Eri II is bound to the Milky Way, we derived the escape velocity at the location of Eri II $(\sim 370 \mathrm{kpc})$ by modeling the dark matter halo of the Milky Way as a Navarro-Frenk-White (NFW) profile (Navarro et al. 1996) with a virial mass of $M_{\mathrm{vir}}=10^{12} M_{\odot}$ and a

\footnotetext{
39 We adopted the circular orbital velocity of the Milky Way at the solar radius $\Theta_{0}=218 \mathrm{~km} \mathrm{~s}^{-1}$ (Bovy et al. 2012) and solar motion of $\left(U_{\odot}, V_{\odot}, W_{\odot}\right)=(11.1,12.24,7.25) \mathrm{km} \mathrm{s}^{-1}$ (Schönrich et al. 2010) for the velocity transformation from heliocentric to the Galactic standard of rest.
} 


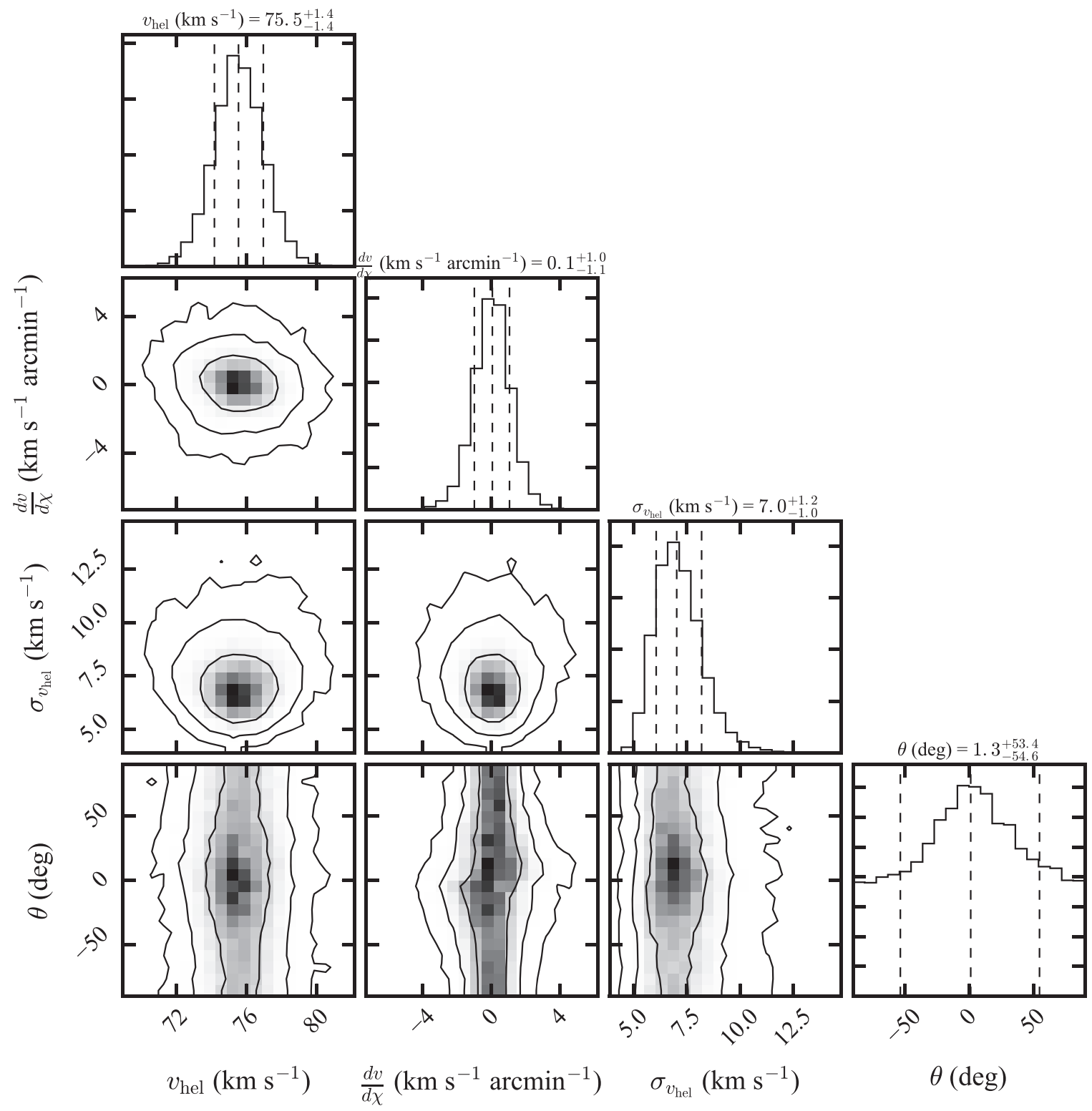

Figure 7. Two-dimensional and marginalized posterior probability distribution from a MCMC sampler using the four-parameter likelihood model defined in the text. The four parameters are mean velocity $v_{\text {hel }}\left[\mathrm{km} \mathrm{s}^{-1}\right]$, velocity dispersion $\sigma_{v \text { hel }}\left[\mathrm{km} \mathrm{s}^{-1}\right]$, velocity gradient $\frac{d v}{d \chi}\left[\mathrm{km} \mathrm{s}^{-1} \operatorname{arcmin}^{-1}\right]$, and position angle of the gradient $\theta$ [deg]. Dashed lines and contours have the same meaning as in Figure 6. We conclude from this analysis that there is no evidence of a velocity gradient in Eri II.

concentration of $c=12$. We find that the escape velocity at the location of Eri II is $\sim 200 \mathrm{~km} \mathrm{~s}^{-1}$ and that Eri II is very likely bound to the Milky Way. While it is possible that Eri II has a very high tangential velocity $\left(\gtrsim 190 \mathrm{~km} \mathrm{~s}^{-1}\right)$, we find that this situation is unlikely based on the results of simulations discussed below.

To infer the orbital parameters and infall time of Eri II, we searched for Eri II analogs in Exploring the Local Volume in Simulations (ELVIS; Garrison-Kimmel et al. 2014), which is a suite of cosmological zoom-in $N$-body simulations using a WMAP7 (Komatsu et al. 2011) $\Lambda$ CDM cosmology. ELVIS includes 24 isolated dark matter halos of masses similar to the Milky Way $\left(M_{\mathrm{vir}}=1.0-2.8 \times 10^{12} M_{\odot}\right.$ and $R_{\mathrm{vir}}=$ $260-360 \mathrm{kpc}){ }^{40}$

\footnotetext{
Note that ELVIS has a total of 48 host halos, and the other 24 halos are in pairs that resemble the masses, distance, and relative velocity of the Milky Way -Andromeda pair. We did not use these paired halos in this analysis.
}

We selected Eri II analogs that are similar to Eri II in both the Galactocentric distance and line-of-sight velocity at redshift $z=0$ and are not located inside of any other halos. In order to calculate the line-of-sight velocity, we approximated the observer as being at the center of the host halo. This is a fair approximation as the Galactocentric distance for Eri II is much larger than Sun's distance to the Milky Way center. Among the 24 ELVIS halos, we found 58 subhalos that possess a similar Galactocentric distance (between 320 and $420 \mathrm{kpc}$ ) and a similar $v_{\mathrm{GSR}}$ (between -85 and $-50 \mathrm{~km} \mathrm{~s}^{-1}$ ) to Eri II and have a stellar mass in the range of $M_{*}=6 \times 10^{3}-10^{5} M_{\odot} \cdot{ }^{41}$ All 58 of these Eri II analog subhalos have a binding energy higher than the kinetic energy, and therefore they are all bound to their

\footnotetext{
${ }^{41}$ Stellar masses in the ELVIS simulations are derived from an abundancematching relation and might have large uncertainties (Garrison-Kimmel et al. 2014). We therefore accepted a large stellar mass range for the Eri II analogs.
} 
Table 1

Summary of the Properties of Eridanus II

\begin{tabular}{|c|c|c|}
\hline Row & Quantity & Value \\
\hline (1) & R.A. (J2000) & 03:44:20.1 \\
\hline (2) & Decl. (J2000) & $-43: 32: 01.7$ \\
\hline (3) & Heliocentric Distance (kpc) & $366 \pm 17$ \\
\hline (4) & Galactocentric Distance (kpc) & $368 \pm 17$ \\
\hline (5) & $M_{V, 0}$ & $-7.1 \pm 0.3$ \\
\hline (6) & $L_{V, 0}\left(L_{\odot}\right)$ & $5.9_{-1.4}^{+1.9} \times 10^{4}$ \\
\hline (7) & $r_{1 / 2}(\mathrm{pc})$ & $277 \pm 14$ \\
\hline (8) & $r_{1 / 2}(\operatorname{arcmin})$ & $2.31 \pm 0.12$ \\
\hline (9) & $\epsilon$ & $0.48 \pm 0.04$ \\
\hline (10) & PA (N to $\mathrm{E} ;$ deg) & $72.6 \pm 3.3$ \\
\hline (11) & $v_{\text {hel }}\left(\mathrm{km} \mathrm{s}^{-1}\right)$ & $75.6 \pm 1.3 \pm 2.0$ \\
\hline (12) & $v_{\mathrm{GSR}}\left(\mathrm{km} \mathrm{s}^{-1}\right)$ & -66.6 \\
\hline (13) & $\sigma_{v}\left(\mathrm{~km} \mathrm{~s}^{-1}\right)$ & $6.9_{-0.9}^{+1.2}$ \\
\hline (14) & $M_{\text {half }}\left(M_{\odot}\right)$ & $1.2_{-0.3}^{+0.4} \times 10^{7}$ \\
\hline (15) & $M / L_{V}\left(M_{\odot} / L_{\odot}\right)$ & $420_{-140}^{+210}$ \\
\hline (16) & $\frac{d v}{d \chi}\left(\mathrm{km} \mathrm{s}^{-1} \operatorname{arcmin}^{-1}\right)$ & $0.1 \pm 1.1$ \\
\hline (17) & Mean metallicity & $-2.38 \pm 0.13$ \\
\hline (18) & Metallicity dispersion (dex) & $0.47_{-0.09}^{+0.12}$ \\
\hline (19) & $\log _{10} J(0 \bullet 2)\left(\mathrm{GeV}^{2} \mathrm{~cm}^{-5}\right)$ & $16.5 \pm 0.8$ \\
\hline (20) & $\log _{10} J(0 \bullet 5)\left(\mathrm{GeV}^{2} \mathrm{~cm}^{-5}\right)$ & $16.6 \pm 0.9$ \\
\hline
\end{tabular}

Note. Rows (1)-(10) are taken or derived from Crnojević et al. (2016). Values in rows (11)-(20) are derived using the measurements in this paper. All values reported here (and in this paper) are from the 50th percentile of the posterior probability distributions. The uncertainties are from the 16th and 84th percentiles of the posterior probability distributions.

host halos. We therefore conclude that Eri II is very likely bound to the Milky Way. Nine of these 58 subhalos are on their first infall (i.e., the subhalos have never entered the virial radius of the host), 41 are on their second passages (i.e., the subhalos just passed the apocenter for the first time) with an infall time of about 4-7 Gyr ago, and 8 are on their third passages (i.e., just passed the apocenter for the second time) with an infall time of 8-10 Gyr ago. The second passage cases can additionally be divided into two categories: 12 subhalos have low-eccentricity orbits with a pericenter $>200 \mathrm{kpc}$ and an orbital period of $\sim 6-7 \mathrm{Gyr}$, while 29 subhalos have higheccentricity orbits with a pericenter $<200 \mathrm{kpc}$ and an orbital period of 4-6 Gyr. We therefore conclude that Eri II is most likely on its second passage, with an eccentric orbit and a firstinfall time of $\sim 5 \mathrm{Gyr}$ ago (with a probability of $\sim 50 \%$ ). Examples of the infall history in each category are given in Figure 8.

\subsection{Star Formation in Eridanus II}

Koposov et al. (2015) noted the presence of seven bright ( $g \sim 20.5$ ) blue stars near the center of Eri II, with colors and magnitudes consistent with being blue-loop stars from a $\sim 250 \mathrm{Myr}$ old population in Eri II (see the stars around $g-r \sim 0.3$ and $g \sim 20.5$ in the left panel of Figure 4). We obtained spectra of five of these stars, and our measurements show that none of them have a velocity close to the systemic velocity of Eri II. We therefore conclude that the location of these stars near Eri II is a coincidence and that there is no evidence of recent star formation. This result is consistent with the star formation history derived by Crnojević et al. (2016) and also with the low H I gas content measured by Westmeier et al. (2015) and Crnojević et al. (2016). In addition to the possible young population, Crnojević et al. (2016) also identified a possible intermediate-age $(3 \mathrm{Gyr})$ population in Eri II. However, our spectroscopic measurements are not deep enough to target any of these stars.

As noted by many previous studies, the dwarf galaxies around the virial radius of the Milky Way show a sharp transition in star formation rate and cold gas content, with the Magellanic Clouds as the only gas-rich star-forming galaxies inside the Milky Way's virial radius (e.g., Einasto et al. 1974; Blitz \& Robishaw 2000; Grcevich \& Putman 2009; Spekkens et al. 2014). Until now, Leo $T(d \sim 420 \mathrm{kpc})$ has been the closest known low-luminosity dwarf outside the virial radius of the Milky Way. Notably, Leo T retains a significant H I gas reservoir (Irwin et al. 2007; Ryan-Weber et al. 2008) and has signatures of star formation within the past few hundred Myr (de Jong et al. 2008; Weisz et al. 2012), but the large gap in distance between the most distant gas-free objects (Leo I and Leo II at $225-250 \mathrm{kpc}$ ) and Leo T limits its utility in constraining gas loss mechanisms for Milky Way satellites (see Figure 9). Eri II is located in this gap, slightly beyond the virial radius, and has a similar luminosity to Leo $\mathrm{T}$ and a higher dynamical mass. It is therefore striking that Eri II has a much lower gas content and apparently lacks any recent star formation. As suggested by Wetzel et al. (2015), quenching at stellar mass $M_{*}=10^{4-5} M_{\odot}$ may arise from a mix of the host-halo environment and cosmic reionization. As a quiescent dwarf galaxy located close to, but beyond, the virial radius of Milky Way, Eri II is a key object for studying environmental influences on low-mass galaxies and the quenching of star formation in such systems (Weisz et al. 2014; Wetzel et al. 2015; Wheeler et al. 2015; Fillingham et al. 2016).

We compare Eri II with Leo $\mathrm{T}$ by searching for Leo $\mathrm{T}$ analogs in the ELVIS simulations, as we did for Eri II in Section 4.4. The radial velocity of Leo $\mathrm{T}\left(v_{\mathrm{GSR}}=-58 \mathrm{~km} \mathrm{~s}^{-1}\right.$; Simon \& Geha 2007) is similar to that of Eri II. However, as Leo $\mathrm{T}$ is about $50 \mathrm{kpc}$ farther from the Milky Way than Eri II, we found that $\sim 30 \%$ of Leo $\mathrm{T}$ analogs are on their first infall into the Milky Way, compared to only $\sim 15 \%$ of Eri II analogs. If the quenching of Eri II was caused by host-halo interactions, the different orbits and infall histories of Eri II and Leo T could be responsible for their differing star formation histories. If Eri II is indeed on its second orbit around the Galaxy, as suggested from the results of ELVIS simulation, then the gas reservoir of Eri II could have been swept away via ram pressure stripping during its first passage. The quenching timescale for galaxies with stellar mass $M_{*}<10^{7} M_{\odot}$ is shorter than 1.5 Gyr (Fillingham et al. 2015; Wetzel et al. 2015), which matches the possible intermediate-age $(3 \mathrm{Gyr})$ population found by Crnojević et al. (2016) well. However, complete removal of the neutral gas during a single orbit around the Milky Way that likely does not closely approach the Galactic disk would place stringent constraints on the halo gas density at large Galactocentric radii. Future proper motion measurements of Eri II and Leo T from HST will better constrain their orbits, determining their infall histories and their orbital pericenters.

Alternatively, the quenching of Eri II could have been caused by cosmic reionization at high redshift (e.g., Bullock et al. 2001; Brown et al. 2014). Reionization can explain the lack of gas in Eri II even if it is on its first infall. However, in that case, Eri II should not show an extended star formation history or an intermediate-age population. Future deep-imaging 
Table 2

Velocity and Metallicity Measurements for Eridanus II

\begin{tabular}{|c|c|c|c|c|c|c|c|c|c|c|}
\hline ID & $\mathrm{MJD}^{\mathrm{a}}$ & $\begin{array}{l}\text { R.A. } \\
\text { (deg) }\end{array}$ & $\begin{array}{l}\text { Decl. } \\
\text { (deg) }\end{array}$ & $\begin{array}{c}g^{\mathrm{b}} \\
(\mathrm{mag})\end{array}$ & $\begin{array}{c}r^{\mathrm{b}} \\
(\mathrm{mag})\end{array}$ & $\mathrm{S} / \mathrm{N}$ & $\begin{array}{c}v \\
\left(\mathrm{~km} \mathrm{~s}^{-1}\right)\end{array}$ & $\begin{array}{l}\mathrm{EW} \\
(\AA)\end{array}$ & {$[\mathrm{Fe} / \mathrm{H}]$} & MEM \\
\hline DES J034338.10-432550.9 & $\begin{array}{l}57345.7 \\
57312.8\end{array}$ & 55.90874 & -43.43079 & 22.23 & 20.82 & $\begin{array}{r}15.4 \\
7.0\end{array}$ & $\begin{array}{l}17.39 \pm 1.14 \\
14.50 \pm 1.99\end{array}$ & $\begin{array}{l}4.61 \pm 0.50 \\
3.07 \pm 1.80\end{array}$ & $\begin{array}{l}\cdots \\
\ldots\end{array}$ & 0 \\
\hline DES J034340.06-432808.3 & $\begin{array}{l}57345.7 \\
57312.8\end{array}$ & 55.91690 & -43.46898 & 20.14 & 18.82 & $\begin{array}{l}59.9 \\
29.2\end{array}$ & $\begin{array}{l}20.12 \pm 1.01 \\
19.64 \pm 1.24\end{array}$ & $\begin{array}{l}5.85 \pm 0.24 \\
5.90 \pm 0.34\end{array}$ & $\begin{array}{l}\cdots \\
\ldots\end{array}$ & 0 \\
\hline DES J034341.56-432918.2 & $\begin{array}{l}57345.7 \\
57312.8\end{array}$ & 55.92317 & -43.48838 & 19.97 & 18.45 & $\begin{array}{r}139.8 \\
70.2\end{array}$ & $\begin{array}{l}52.41 \pm 1.00 \\
54.07 \pm 1.21\end{array}$ & $\begin{array}{l}2.15 \pm 0.20 \\
2.30 \pm 0.21\end{array}$ & $\begin{array}{l}\cdots \\
\ldots\end{array}$ & 0 \\
\hline DES J034343.30-432810.1 & $\begin{array}{l}57345.7 \\
57312.8\end{array}$ & 55.93042 & -43.46948 & 20.63 & 19.22 & $\begin{array}{l}68.4 \\
32.4\end{array}$ & $\begin{array}{l}13.19 \pm 1.01 \\
13.89 \pm 1.23\end{array}$ & $\begin{array}{l}3.55 \pm 0.23 \\
3.50 \pm 0.32\end{array}$ & $\begin{array}{l}\ldots \\
\ldots\end{array}$ & 0 \\
\hline DES J034347.77-432951.7 & $\begin{array}{l}57345.7 \\
57312.8\end{array}$ & 55.94904 & -43.49771 & 20.53 & 20.21 & $\begin{array}{r}13.2 \\
5.7\end{array}$ & $\begin{array}{l}306.12 \pm 1.27 \\
308.51 \pm 2.30\end{array}$ & $\begin{array}{c}4.43 \pm 0.45 \\
\ldots\end{array}$ & $\begin{array}{l}\ldots \\
\ldots\end{array}$ & 0 \\
\hline DES J034349.44-432432.1 & $\begin{array}{l}57345.7 \\
57312.8\end{array}$ & 55.95601 & -43.40893 & 20.23 & 18.85 & $\begin{array}{l}69.9 \\
33.8\end{array}$ & $\begin{array}{l}105.33 \pm 1.01 \\
103.55 \pm 1.26\end{array}$ & $\begin{array}{l}5.05 \pm 0.23 \\
5.55 \pm 0.31\end{array}$ & $\begin{array}{l}\ldots \\
\ldots\end{array}$ & 0 \\
\hline DES J034349.53-432609.3 & $\begin{array}{l}57345.7 \\
57312.8\end{array}$ & 55.95637 & -43.43590 & 21.58 & 20.48 & $\begin{array}{r}14.8 \\
7.1\end{array}$ & $\begin{array}{l}219.50 \pm 1.42 \\
220.91 \pm 2.02\end{array}$ & $\begin{array}{l}\cdots \\
\ldots\end{array}$ & $\begin{array}{l}\cdots \\
\ldots\end{array}$ & 0 \\
\hline DES J034349.54-432951.6 & $\begin{array}{l}57345.7 \\
57312.8\end{array}$ & 55.95642 & -43.49765 & 20.64 & 19.17 & $\begin{array}{l}75.9 \\
37.9\end{array}$ & $\begin{array}{l}42.94 \pm 1.01 \\
44.60 \pm 1.22\end{array}$ & $\begin{array}{l}3.82 \pm 0.22 \\
3.68 \pm 0.30\end{array}$ & $\begin{array}{l}\ldots \\
\ldots\end{array}$ & 0 \\
\hline DES J034351.43-432639.7 & $\begin{array}{l}57345.7 \\
57312.8\end{array}$ & 55.96431 & -43.44436 & 21.74 & 20.25 & $\begin{array}{l}26.8 \\
11.7\end{array}$ & $\begin{array}{l}-2.63 \pm 1.04 \\
-3.70 \pm 1.46\end{array}$ & $\begin{array}{l}4.54 \pm 0.22 \\
4.76 \pm 0.66\end{array}$ & $\begin{array}{l}\cdots \\
\ldots\end{array}$ & 0 \\
\hline DES J034351.50-432714.9 & $\begin{array}{l}57345.7 \\
57312.8\end{array}$ & 55.96460 & -43.45415 & 19.55 & 18.94 & $\begin{array}{l}37.6 \\
17.9\end{array}$ & $\begin{array}{l}46.49 \pm 1.02 \\
49.18 \pm 1.31\end{array}$ & $\begin{array}{c}\ldots \\
7.14 \pm 0.51\end{array}$ & $\begin{array}{l}\ldots \\
\ldots\end{array}$ & 0 \\
\hline DES J034355.42-432447.2 & $\begin{array}{l}57345.7 \\
57312.8\end{array}$ & 55.98091 & -43.41310 & 20.36 & 20.12 & $\begin{array}{r}14.3 \\
7.1\end{array}$ & $\begin{array}{l}150.44 \pm 1.60 \\
147.56 \pm 2.13\end{array}$ & $\begin{array}{l}2.66 \pm 0.50 \\
2.56 \pm 0.59\end{array}$ & $\begin{array}{l}\cdots \\
\ldots\end{array}$ & 0 \\
\hline DES J034402.04-432608.5 & $\begin{array}{l}57345.7 \\
57312.8\end{array}$ & 56.00848 & -43.43570 & 20.98 & 19.56 & $\begin{array}{l}63.8 \\
30.3\end{array}$ & $\begin{array}{l}83.30 \pm 1.01 \\
82.90 \pm 1.24\end{array}$ & $\begin{array}{l}3.72 \pm 0.23 \\
3.78 \pm 0.36\end{array}$ & $\begin{array}{l}\ldots \\
\ldots\end{array}$ & 0 \\
\hline DES J034402.24-433158.8 & 57345.7 & 56.00932 & -43.53299 & 21.73 & 21.00 & 7.2 & $69.80 \pm 1.61$ & $4.92 \pm 0.77$ & $-1.85 \pm 0.31$ & 1 \\
\hline DES J034404.78-432727.7 & $\begin{array}{l}57345.7 \\
57312.8\end{array}$ & 56.01991 & -43.45769 & 21.07 & 20.12 & $\begin{array}{r}20.0 \\
8.1\end{array}$ & $\begin{array}{l}64.87 \pm 1.43 \\
67.53 \pm 3.08\end{array}$ & $\begin{array}{l}\cdots \\
\ldots\end{array}$ & $\begin{array}{l}\cdots \\
\ldots\end{array}$ & 0 \\
\hline DES J034406.25-432811.1 & 57345.7 & 56.02605 & -43.46976 & 22.34 & 21.07 & 11.4 & $116.91 \pm 1.57$ & $\ldots$ & $\ldots$ & 0 \\
\hline DES J034406.86-433105.2 & 57345.7 & 56.02857 & -43.51812 & 22.86 & 21.68 & 5.9 & $262.86 \pm 2.84$ & $\ldots$ & $\ldots$ & 0 \\
\hline DES J034406.94-433143.4 & $\begin{array}{l}57345.7 \\
57312.8\end{array}$ & 56.02892 & -43.52871 & 21.11 & 20.16 & $\begin{array}{r}15.2 \\
7.2\end{array}$ & $\begin{array}{l}77.77 \pm 1.23 \\
77.96 \pm 1.81\end{array}$ & $\begin{array}{l}\cdots \\
\ldots\end{array}$ & $\begin{array}{l}\cdots \\
\ldots\end{array}$ & 1 \\
\hline DES J034408.52-433046.8 & $\begin{array}{l}57345.7 \\
57312.8\end{array}$ & 56.03551 & -43.51300 & 20.00 & 18.77 & $\begin{array}{l}61.3 \\
28.9\end{array}$ & $\begin{array}{l}18.78 \pm 1.01 \\
21.47 \pm 1.24\end{array}$ & $\begin{array}{l}5.67 \pm 0.24 \\
5.23 \pm 0.34\end{array}$ & $\begin{array}{l}\ldots \\
\ldots\end{array}$ & 0 \\
\hline DES J034410.56-432602.0 & $\begin{array}{l}57345.7 \\
57312.8\end{array}$ & 56.04402 & -43.43390 & 20.05 & 18.65 & $\begin{array}{l}97.6 \\
47.4\end{array}$ & $\begin{array}{l}39.08 \pm 1.00 \\
40.66 \pm 1.21\end{array}$ & $\begin{array}{l}4.26 \pm 0.22 \\
4.27 \pm 0.26\end{array}$ & $\begin{array}{l}\ldots \\
\ldots\end{array}$ & 0 \\
\hline DES J034411.10-433052.1 & 57345.7 & 56.04626 & -43.51447 & 22.02 & 21.28 & 6.5 & $65.37 \pm 2.30$ & $\ldots$ & $\ldots$ & 1 \\
\hline DES J034412.28-433105.9 & 57345.7 & 56.05116 & -43.51831 & 21.98 & 21.25 & 5.3 & $75.12 \pm 2.57$ & $\ldots$ & $\ldots$ & 1 \\
\hline DES J034412.37-432803.5 & 57345.7 & 56.05156 & -43.46764 & 21.44 & 20.64 & 12.5 & $308.95 \pm 1.20$ & $\ldots$ & $\ldots$ & 0 \\
\hline DES J034412.63-433031.3 & $\begin{array}{l}57345.7 \\
57312.8\end{array}$ & 56.05264 & -43.50870 & 21.79 & 21.10 & $\begin{array}{l}8.2 \\
3.4\end{array}$ & $\begin{array}{l}91.01 \pm 1.94 \\
91.67 \pm 2.62\end{array}$ & $\begin{array}{l}\cdots \\
\ldots\end{array}$ & $\begin{array}{l}\ldots \\
\ldots\end{array}$ & 1 \\
\hline DES J034414.62-433134.8 & $\begin{array}{l}57345.7 \\
57312.8\end{array}$ & 56.06092 & -43.52633 & 21.57 & 20.68 & $\begin{array}{r}13.0 \\
5.7\end{array}$ & $\begin{array}{l}72.37 \pm 1.54 \\
73.94 \pm 2.08\end{array}$ & $\begin{array}{c}3.35 \pm 0.49 \\
\ldots\end{array}$ & $\begin{array}{c}-2.54 \pm 0.21 \\
\ldots\end{array}$ & 1 \\
\hline DES J034415.65-433032.0 & 57345.7 & 56.06520 & -43.50890 & 22.09 & 21.31 & 7.4 & $65.75 \pm 1.60$ & $\ldots$ & $\ldots$ & 1 \\
\hline DES J034416.14-433243.4 & $\begin{array}{l}57345.7 \\
57312.8\end{array}$ & 56.06724 & -43.54538 & 20.58 & 19.58 & $\begin{array}{l}28.8 \\
14.2\end{array}$ & $\begin{array}{l}74.11 \pm 1.05 \\
73.94 \pm 1.36\end{array}$ & $\begin{array}{l}3.96 \pm 0.26 \\
4.25 \pm 0.51\end{array}$ & $\begin{array}{l}-2.54 \pm 0.12 \\
-2.43 \pm 0.20\end{array}$ & 1 \\
\hline DES J034416.29-433038.7 & 57345.7 & 56.06786 & -43.51076 & 20.49 & 20.15 & 13.4 & $199.69 \pm 1.46$ & $2.96 \pm 0.60$ & $\ldots$ & 0 \\
\hline DES J034418.18-433111.9 & $\begin{array}{l}57345.7 \\
57312.8\end{array}$ & 56.07574 & -43.51998 & 21.40 & 20.69 & $\begin{array}{r}10.8 \\
4.3\end{array}$ & $\begin{array}{l}80.37 \pm 1.70 \\
81.44 \pm 3.16\end{array}$ & $\begin{array}{c}3.19 \pm 0.50 \\
\ldots\end{array}$ & $\begin{array}{c}-2.63 \pm 0.22 \\
\ldots\end{array}$ & 1 \\
\hline DES J034419.20-433018.9 & 57345.7 & 56.08000 & -43.50525 & 22.36 & 21.68 & 5.4 & $81.69 \pm 2.95$ & $\ldots$ & $\ldots$ & 1 \\
\hline DES J034420.20-433210.9 & $\begin{array}{l}57345.7 \\
57312.8\end{array}$ & 56.08417 & -43.53636 & 21.42 & 20.53 & $\begin{array}{r}12.0 \\
6.3\end{array}$ & $\begin{array}{l}76.71 \pm 1.62 \\
78.55 \pm 2.14\end{array}$ & $\begin{array}{l}\cdots \\
\ldots\end{array}$ & $\begin{array}{l}\cdots \\
\ldots\end{array}$ & 1 \\
\hline DES J034420.62-433308.1 & $\begin{array}{l}57345.7 \\
57312.8\end{array}$ & 56.08593 & -43.55225 & 21.71 & 21.02 & $\begin{array}{l}7.4 \\
3.9\end{array}$ & $\begin{array}{l}84.54 \pm 2.04 \\
79.33 \pm 3.24\end{array}$ & $\begin{array}{l}\ldots \\
\ldots\end{array}$ & $\begin{array}{l}\ldots \\
\ldots\end{array}$ & 1 \\
\hline DES J034420.77-433227.6 & $\begin{array}{l}57345.7 \\
57312.8\end{array}$ & 56.08655 & -43.54101 & 21.28 & 20.37 & $\begin{array}{r}16.8 \\
7.6\end{array}$ & $\begin{array}{l}86.55 \pm 1.60 \\
85.82 \pm 2.02\end{array}$ & $\begin{array}{l}5.06 \pm 0.49 \\
5.05 \pm 0.75\end{array}$ & $\begin{array}{l}-1.94 \pm 0.20 \\
-1.94 \pm 0.29\end{array}$ & 1 \\
\hline DES J034421.34-433020.9 & $\begin{array}{l}57345.7 \\
57312.8\end{array}$ & 56.08892 & -43.50581 & 21.30 & 20.43 & $\begin{array}{r}15.3 \\
6.7\end{array}$ & $\begin{array}{l}75.80 \pm 1.19 \\
83.36 \pm 2.42\end{array}$ & $\begin{array}{c}5.07 \pm 0.52 \\
\ldots\end{array}$ & $\begin{array}{c}-1.92 \pm 0.21 \\
\ldots\end{array}$ & 1 \\
\hline DES J034423.06-433124.4 & $\begin{array}{l}57345.7 \\
57312.8\end{array}$ & 56.09608 & -43.52345 & 21.67 & 20.94 & $\begin{array}{l}9.5 \\
3.6\end{array}$ & $\begin{array}{l}74.74 \pm 1.41 \\
72.81 \pm 2.42\end{array}$ & $\begin{array}{c}2.63 \pm 0.60 \\
\ldots\end{array}$ & $\begin{array}{c}-2.82 \pm 0.28 \\
\ldots\end{array}$ & 1 \\
\hline DES J034423.98-433243.6 & $\begin{array}{l}57345.7 \\
57312.8\end{array}$ & 56.09990 & -43.54543 & 21.05 & 20.09 & $\begin{array}{r}19.9 \\
9.7\end{array}$ & $\begin{array}{l}67.60 \pm 1.12 \\
67.79 \pm 1.53\end{array}$ & $\begin{array}{l}4.60 \pm 0.34 \\
3.64 \pm 0.44\end{array}$ & $\begin{array}{l}-2.18 \pm 0.14 \\
-2.55 \pm 0.18\end{array}$ & 1 \\
\hline DES J034426.53-433243.9 & $\begin{array}{l}57345.7 \\
57312.8\end{array}$ & 56.11054 & -43.54552 & 21.29 & 20.58 & $\begin{array}{r}12.3 \\
4.7\end{array}$ & $\begin{array}{l}68.82 \pm 1.34 \\
71.50 \pm 2.21\end{array}$ & $\begin{array}{c}5.44 \pm 0.55 \\
\ldots\end{array}$ & $\begin{array}{c}-1.76 \pm 0.22 \\
\ldots\end{array}$ & 1 \\
\hline
\end{tabular}


Table 2

(Continued)

\begin{tabular}{|c|c|c|c|c|c|c|c|c|c|c|}
\hline ID & $\mathrm{MJD}^{\mathrm{a}}$ & $\begin{array}{l}\text { R.A. } \\
\text { (deg) }\end{array}$ & $\begin{array}{l}\text { Decl. } \\
(\mathrm{deg})\end{array}$ & $\begin{array}{c}g^{\mathrm{b}} \\
(\mathrm{mag})\end{array}$ & $\begin{array}{c}r^{\mathrm{b}} \\
(\mathrm{mag})\end{array}$ & $\mathrm{S} / \mathrm{N}$ & $\begin{array}{c}v \\
\left(\mathrm{~km} \mathrm{~s}^{-1}\right)\end{array}$ & $\begin{array}{l}\mathrm{EW} \\
(\AA)\end{array}$ & {$[\mathrm{Fe} / \mathrm{H}]$} & MEM \\
\hline DES J034426.64-433122.8 & $\begin{array}{l}57345.7 \\
57312.8\end{array}$ & 56.11100 & -43.52301 & 21.18 & 20.24 & $\begin{array}{r}19.7 \\
8.2\end{array}$ & $\begin{array}{l}77.32 \pm 1.19 \\
79.47 \pm 1.60\end{array}$ & $\begin{array}{c}5.37 \pm 0.40 \\
\ldots\end{array}$ & $\begin{array}{c}-1.85 \pm 0.16 \\
\ldots\end{array}$ & 1 \\
\hline DES J034427.50-433252.8 & 57345.7 & 56.11457 & -43.54801 & 21.98 & 21.34 & 5.1 & $75.07 \pm 2.40$ & $4.07 \pm 0.51$ & $-2.12 \pm 0.22$ & 1 \\
\hline DES J034428.27-433250.7 & $\begin{array}{l}57345.7 \\
57312.8\end{array}$ & 56.11778 & -43.54742 & 20.75 & 19.77 & $\begin{array}{l}26.9 \\
12.4\end{array}$ & $\begin{array}{l}72.18 \pm 1.05 \\
71.13 \pm 1.42\end{array}$ & $\begin{array}{l}3.78 \pm 0.31 \\
3.46 \pm 0.50\end{array}$ & $\begin{array}{l}-2.57 \pm 0.13 \\
-2.69 \pm 0.21\end{array}$ & 1 \\
\hline DES J034429.32-433130.3 & $\begin{array}{l}57345.7 \\
57312.8\end{array}$ & 56.12217 & -43.52509 & 21.41 & 20.71 & $\begin{array}{r}11.3 \\
5.2\end{array}$ & $\begin{array}{l}73.91 \pm 1.27 \\
78.05 \pm 2.59\end{array}$ & $\begin{array}{c}3.12 \pm 0.26 \\
\ldots\end{array}$ & $\begin{array}{c}-2.66 \pm 0.12 \\
\ldots\end{array}$ & 1 \\
\hline DES J034429.71-433147.9 & $\begin{array}{l}57345.7 \\
57312.8\end{array}$ & 56.12379 & -43.52998 & 21.17 & 20.32 & $\begin{array}{r}17.1 \\
7.9\end{array}$ & $\begin{array}{l}70.79 \pm 1.21 \\
72.21 \pm 1.67\end{array}$ & $\begin{array}{c}1.65 \pm 0.22 \\
\ldots\end{array}$ & $\begin{array}{c}-3.42 \pm 0.15 \\
\ldots\end{array}$ & 1 \\
\hline DES J034430.00-433305.8 & 57345.7 & 56.12499 & -43.55162 & 20.51 & 20.19 & 14.4 & $224.86 \pm 1.46$ & $\ldots$ & $\ldots$ & 0 \\
\hline DES J034430.24-433048.0 & 57345.7 & 56.12601 & -43.51333 & 22.41 & 21.75 & 4.6 & $79.29 \pm 2.40$ & $\ldots$ & $\ldots$ & 1 \\
\hline DES J034431.11-433316.0 & $\begin{array}{l}57345.7 \\
57312.8\end{array}$ & 56.12962 & -43.55444 & 21.70 & 20.93 & $\begin{array}{l}9.0 \\
4.5\end{array}$ & $\begin{array}{l}89.73 \pm 1.52 \\
89.41 \pm 3.03\end{array}$ & $\begin{array}{c}4.49 \pm 0.77 \\
\ldots\end{array}$ & $\begin{array}{c}-2.04 \pm 0.31 \\
\ldots\end{array}$ & 1 \\
\hline DES J034433.36-433319.1 & 57345.7 & 56.13898 & -43.55531 & 21.84 & 21.08 & 7.0 & $66.71 \pm 1.98$ & $\ldots$ & $\ldots$ & 1 \\
\hline DES J034435.17-433306.2 & $\begin{array}{l}57345.7 \\
57312.8\end{array}$ & 56.14654 & -43.55172 & 22.39 & 21.00 & $\begin{array}{r}16.2 \\
7.2\end{array}$ & $\begin{array}{l}72.36 \pm 1.37 \\
72.16 \pm 1.69\end{array}$ & $\begin{array}{l}4.54 \pm 0.47 \\
4.06 \pm 0.60\end{array}$ & $\begin{array}{l}\ldots \\
\ldots\end{array}$ & 0 \\
\hline DES J034437.06-433420.7 & $\begin{array}{l}57345.7 \\
57312.8\end{array}$ & 56.15440 & -43.57242 & 22.69 & 21.31 & $\begin{array}{r}14.6 \\
7.2\end{array}$ & $\begin{array}{l}14.30 \pm 1.16 \\
11.95 \pm 1.65\end{array}$ & $\begin{array}{c}4.51 \pm 0.56 \\
\ldots\end{array}$ & $\begin{array}{l}\ldots \\
\ldots\end{array}$ & 0 \\
\hline DES J034437.87-433457.2 & 57345.7 & 56.15777 & -43.58255 & 21.82 & 21.04 & 5.6 & $362.64 \pm 2.80$ & $\cdots$ & $\cdots$ & 0 \\
\hline DES J034438.15-433549.0 & $\begin{array}{l}57345.7 \\
57312.8\end{array}$ & 56.15897 & -43.59693 & 20.17 & 18.75 & $\begin{array}{l}71.7 \\
37.9\end{array}$ & $\begin{array}{l}-1.20 \pm 1.01 \\
-1.43 \pm 1.22\end{array}$ & $\begin{array}{l}4.38 \pm 0.22 \\
4.59 \pm 0.28\end{array}$ & $\begin{array}{l}\cdots \\
\ldots\end{array}$ & 0 \\
\hline DES J034438.78-433015.2 & $\begin{array}{l}57345.7 \\
57312.8\end{array}$ & 56.16156 & -43.50421 & 21.79 & 21.06 & $\begin{array}{l}8.5 \\
2.6\end{array}$ & $\begin{array}{l}73.76 \pm 2.09 \\
75.78 \pm 3.89\end{array}$ & $\begin{array}{l}\ldots \\
\ldots\end{array}$ & $\begin{array}{l}\ldots \\
\ldots\end{array}$ & 1 \\
\hline DES J034439.68-433038.6 & 57345.7 & 56.16534 & -43.51073 & 21.94 & 21.20 & 5.9 & $68.39 \pm 2.17$ & $\ldots$ & $\ldots$ & 1 \\
\hline DES J034440.26-433419.3 & $\begin{array}{l}57345.7 \\
57312.8\end{array}$ & 56.16773 & -43.57202 & 21.26 & 19.89 & $\begin{array}{l}34.8 \\
17.9\end{array}$ & $\begin{array}{l}49.29 \pm 1.04 \\
52.49 \pm 1.32\end{array}$ & $\begin{array}{l}5.00 \pm 0.29 \\
5.05 \pm 0.54\end{array}$ & $\begin{array}{l}\ldots \\
\ldots\end{array}$ & 0 \\
\hline DES J034440.32-433336.5 & 57345.7 & 56.16801 & -43.56015 & 20.46 & 20.26 & 11.4 & $-120.75 \pm 1.41$ & $\ldots$ & $\ldots$ & 0 \\
\hline DES J034445.57-432955.4 & $\begin{array}{l}57345.7 \\
57312.8\end{array}$ & 56.18989 & -43.49872 & 21.23 & 20.43 & $\begin{array}{r}16.2 \\
7.1\end{array}$ & $\begin{array}{l}82.22 \pm 1.24 \\
76.41 \pm 1.87\end{array}$ & $\begin{array}{c}2.20 \pm 0.39 \\
\ldots\end{array}$ & $\begin{array}{c}-3.12 \pm 0.20 \\
\ldots\end{array}$ & 1 \\
\hline
\end{tabular}

Notes.

${ }^{\mathrm{a}} \mathrm{MJD}=57345.7$ corresponds to the November run and MJD $=57312.8$ corresponds to the October run. Since for both runs the observations were made over two nights, the MJD listed here is the weighted mean observation date, which occurs during daylight hours.

b Quoted magnitudes represent the weighted-average dereddened PSF magnitude derived from the DES images with SExtractor.

data from $H S T$ will help determine whether star formation in Eri II ended very early, as in other ultra-faint dwarfs, or continued to later times. We note that Eri II is more distant and more massive than any of the Milky Way satellites that are strongly suspected to be quenched by reionization based on previous deep HST imaging (Brown et al. 2014). In this scenario, the key question is why Eri II was more susceptible to the effects of reionization than Leo $\mathrm{T}$, which is currently less massive than Eri II but still contains gas. One possible explanation for the contrast between the observed properties of the two systems is that Leo $\mathrm{T}$ was farther away from the proto-Milky Way at the time of reionization. This hypothesis can be tested with proper motion measurements. If Leo $\mathrm{T}$ first fell into the Milky Way much later than Eri II, it is also possible that its isolation allowed a late phase of gas accretion and associated star formation (Ricotti 2009).

\subsection{Constraints on the Nature of Dark Matter}

Ultra-faint dwarf galaxies are ideal targets for understanding the nature of dark matter. They can provide strong tests of models where dark matter is composed of weakly interacting massive particles (WIMPs) that self-annihilate to produce gamma-rays (Gunn et al. 1978; Bergström \& Snellman 1988; Baltz et al. 2008). The predicted gamma-ray signal from annihilation is proportional to the line-of-sight integral through the square of the dark matter density, or so-called $J$-factor. The $J$-factor is derived by modeling the velocity using the spherical Jeans equation with prior assumptions on the parameterization of the dark matter halo profile. (e.g., Strigari et al. 2008; Essig et al. 2009; Charbonnier et al. 2011; Geringer-Sameth et al. 2015; Martinez 2015). Following the procedure of Simon et al. (2015), we model the dark matter halo as a generalized NFW profile (Navarro et al. 1996). We use flat, "uninformative" priors on the dark matter halo parameters (see, e.g., Essig et al. 2009) and assume a constant stellar velocity anisotropy. We find an integrated $J$-factor for Eri II of $\log _{10}(J)=16.5 \pm 0.8$ within solid angle of 0.2 , and $\log _{10}(J)=16.6 \pm 0.9$ within 0.5 . The error bars represent the difference between the 16th and 84th percentiles and the median of the posterior distribution of $J$-factor. These values assume that the dark matter halo extends beyond the radius of the outermost spectroscopically confirmed star, but truncates within the estimated tidal radius for the dark matter halo. Given that Eri II is $\sim 370 \mathrm{kpc}$ away, the tidal radius could extend far beyond its outermost star location. We derive a tidal radius of $\sim 10 \mathrm{kpc}$ following the description in Geringer-Sameth et al. (2015). The $J$-factor of Eri II is $\sim 3$ orders of magnitude smaller than the most promising dwarf galaxies, which is a direct result of the distance between Eri II and the Sun. This value is consistent with the value predicted from a simple distance 


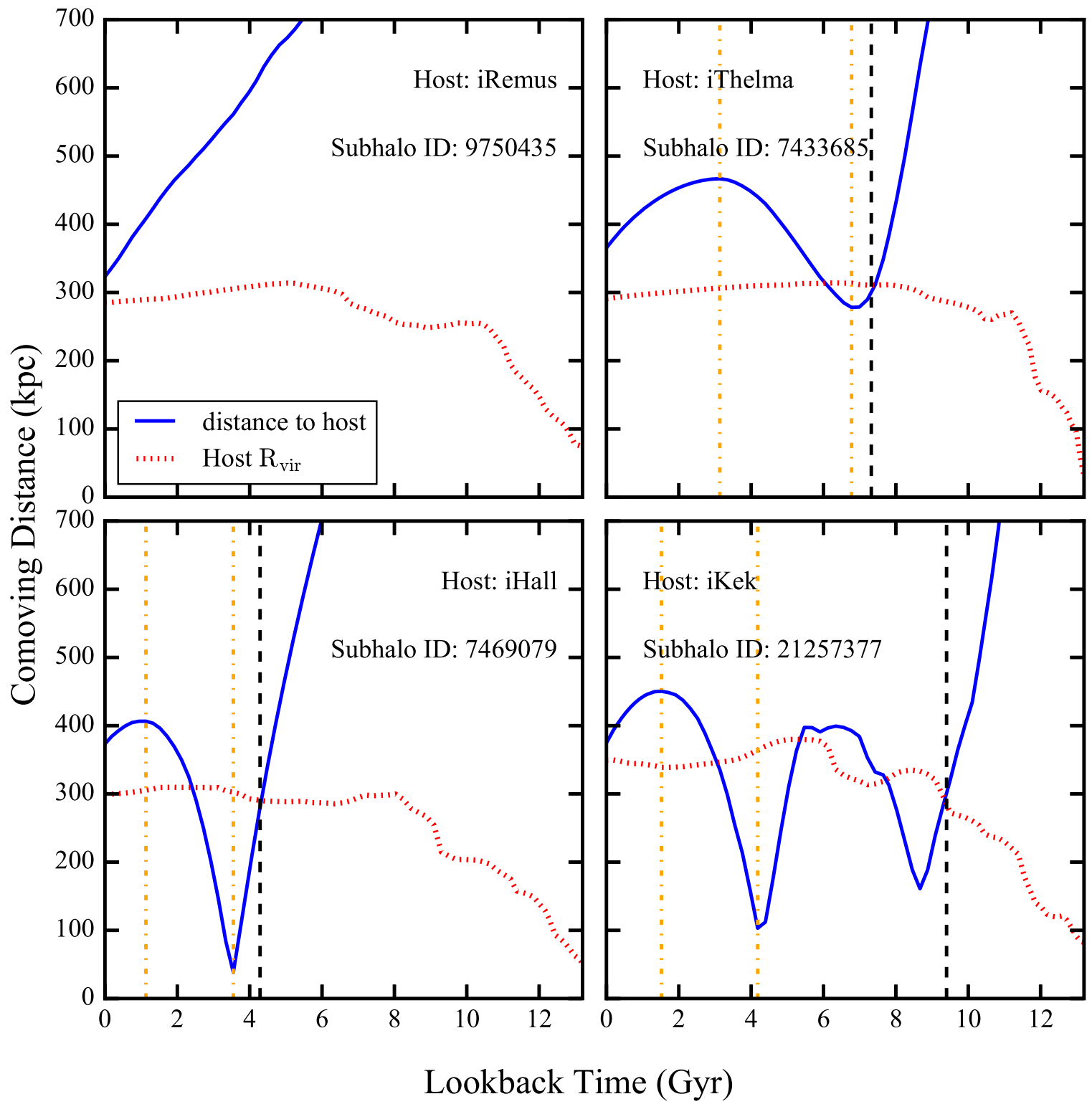

Figure 8. Examples of subhalos in the ELVIS simulations that have distances and velocities similar to Eri II. These subhalos represent four different infall histories for Eri II-first infall (upper left), second passage with low eccentricity (upper right), second passage with high eccentricity (lower left), and third passage (lower right). For each panel, the name of the Milky Way-like host halo and the ID of the subhalo from ELVIS are displayed. The blue solid lines show the (comoving) distance of the subhalo to the host halo and the red dotted lines show the change in the virial radius of the subhalo, both as a function of cosmic time (with time running from right to left). The black dashed lines indicate the infall time of the subhalo and the yellow dashed-dotted lines represent when the subhalo reached its pericenter and apocenter. About half of the Eri II analogs found in ELVIS simulation are on their second passage with high eccentric orbit (i.e., similar to the lower left panel); we conclude that this is the most likely scenario for the Eri II infall history. However, proper motion measurements are necessary to better constrain the orbit of Eri II.

scaling based on the $J$-factors of known dwarfs (e.g., DrlicaWagner et al. 2015a).

While Eri II does not appear to be a promising target for indirect searches for WIMP annihilation because of its distance, the existence of a central star cluster offers a unique opportunity to constrain massive compact halo object (MACHO) dark matter with mass $\gtrsim 10 M_{\odot}$ (Brandt 2016). The recent detection of gravitational waves from the coalescence of $\sim 30 M_{\odot}$ black holes (Abbott et al. 2016) has led to the suggestion that primordial black holes with a similar mass could constitute the dark matter (e.g., Bird et al. 2016; Clesse \& García-Bellido 2016). Interestingly, microlensing and wide binary searches do not exclude MACHOs in the mass range between $20 M_{\odot}$ and $100 M_{\odot}$ (e.g., Alcock et al. 2001; Tisserand et al. 2007; Quinn et al. 2009). We therefore examine the constraints that can be placed on MACHO dark matter using the measured properties of Eri II.

Brandt (2016) argued that MACHO dark matter would dynamically heat, and eventually dissolve, the star cluster near the center of Eri II. Brandt projected MACHO constraints from the survival of this star cluster assuming several values for the 3D velocity dispersion, $\sigma_{3 \mathrm{D}}$, and dark matter density, $\rho$, of Eri II. However, the kinematics measured in Section 4.1 allowed us to directly derive the $3 \mathrm{D}$ velocity dispersion and dark matter density of Eri II: $\sigma_{3 \mathrm{D}} \sim 12 \mathrm{~km} \mathrm{~s}^{-1}$ and $\rho \sim 0.15 M_{\odot} \mathrm{pc}^{-3},{ }^{42}$ assuming a uniform and isotropic distribution of dark matter within the half-light radius. With these

\footnotetext{
$\overline{42}$ Here we assume that velocity dispersion for the dark matter halo is the same as the velocity dispersion for the stars, and $\sigma_{3 \mathrm{D}}=\sqrt{3} \sigma_{1 \mathrm{D}}$.
} 


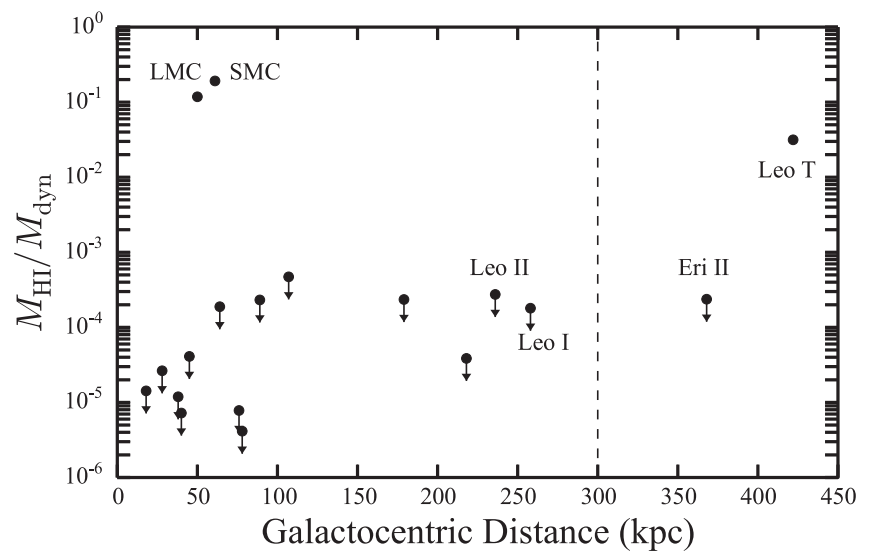

Figure 9. Ratio of H I mass, $M_{\mathrm{HI}}$, to dynamical mass, $M_{\mathrm{dyn}}$, for Milky Way satellites as a function of the Galactocentric distance. The LMC, SMC, and Leo $\mathrm{T}$ are the only Milky Way satellites that have detected neutral gas. The upper limits on $\mathrm{H}$ I mass are used for other satellites. The H I masses (or upper limits) are taken from Crnojević et al. (2016) for Eri II, from Ryan-Weber et al. (2008) for Leo T, from Brüns et al. (2005) for the LMC and SMC, and from Spekkens et al. (2014) for the remaining dwarfs. For the LMC and SMC, the dynamical mass is adopted from van der Marel et al. (2002) and Stanimirović et al. (2004). For the pressure-supported systems, the dynamical mass is computed as the mass enclosed within the half-light radius by adopting the formula from Wolf et al. (2010), using the velocity dispersion and half-light radius from McConnachie (2012) (except for Eri II, which is from this paper) The vertical dashed line shows the approximate virial radius of the Milky Way, $R_{\text {vir }} \sim 300 \mathrm{kpc}$

halo properties, we derived MACHO constraints assuming the stellar cluster has an age of $3 \mathrm{Gyr}$, an initial half-light radius of $r_{h, 0} \sim 13 \mathrm{pc}$, and a mass of $2000 M_{\odot}{ }^{43}$ as shown in Figure 10. When the results from Eri II are combined with those from microlensing and wide binary searches (Alcock et al. 2001; Tisserand et al. 2007; Quinn et al. 2009), MACHOs with mass $\gtrsim 10^{-7} M_{\odot}$ are constrained to be a subdominant component of dark matter. However, if there were an intermediate-mass black hole (IMBH) of mass $M_{\mathrm{BH}}=1500 M_{\odot}$ at the center of Eri II, as extrapolation of the scaling relation from Kruijssen \& Lützgendorf (2013) suggests, its gravity would stabilize the star cluster and would prevent its evaporation. This effect would weaken the bounds and allow for a MACHO mass distribution peaked at a few tens of solar masses to be the main component of the dark matter in the universe (Clesse \& GarcíaBellido 2015).

In addition to constraints on MACHOs, Eri II may also offer an opportunity to constrain the density profile of dark matter halos, addressing the so-called cusp-core problem. Historically, cosmological simulations (Dubinski \& Carlberg 1991; Navarro et al. 1996) predict that dark matter halos should have a cuspy central density profile. However, observational results have consistently pointed to shallower profiles (e.g., de Blok et al. 2001; Walker \& Penarrubia 2011; Adams et al. 2014), and theoretical calculations have been developed to explain why cusps are not found (e.g., Governato et al. 2012; Pontzen \& Governato 2012; Di Cintio et al. 2014). In these models, dark matter cores arise from gravitational interactions between dark matter and baryons, and should occur in dwarf galaxies

\footnotetext{
43 The star cluster has an absolute magnitude of $M_{v}=-3.5\left(\sim 2000 L_{\odot}\right)$ and half-light radius $r_{h \text {,cluster }}=13 \mathrm{pc}$ (Crnojević et al. 2016). The assumptions for the stellar cluster are based on these observational results and the $3 \mathrm{Gyr}$ intermediate-age population found in Eri II (Crnojević et al. 2016). We note that an older population for the cluster is possible, which would lead to a stronger MACHO constraint (i.e., shift the curve leftward).
}

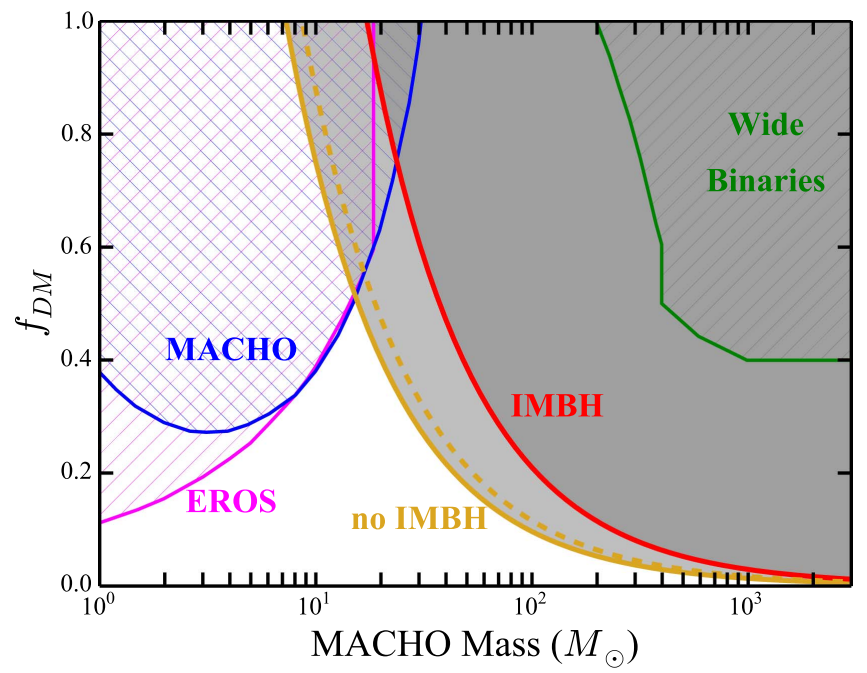

Figure 10. Constraints on MACHO dark matter following the prescription of Brandt (2016), assuming that the Eri II star cluster is located at the center of the Eri II dark matter potential. Colored curves mark exclusion regions for the maximum fraction of dark matter $\left(f_{\mathrm{DM}}\right)$ in MACHOs for a given MACHO mass. The solid yellow curve corresponds to the limits derived from the observed 3D velocity dispersion of $\sigma_{3 D}=12 \mathrm{~km} \mathrm{~s}^{-1}$ and implied central dark matter density of $\rho=0.15 M_{\odot} \mathrm{pc}^{-3}$. As a comparison, the limit derived from $\sigma_{3 D}=8 \mathrm{~km} \mathrm{~s}^{-1}$ and $\rho=0.02 M_{\odot} \mathrm{pc}^{-3}$ from Brandt (2016) is shown as the dashed yellow curve. Since the increase in $\sigma_{3 D}$ loosens the constraint (i.e., shifts the curve rightward) and the increase in $\rho$ strengthens the constraint (i.e., shifts the curve leftward), the combination of the two leads to similar results despite different $\sigma_{3 D}$ and $\rho$ values. The red curve shows the MACHO constraint assuming that an intermediate-mass black hole (IMBH) with mass of $1500 M_{\odot}$ resides at the center of Eri II. Magenta and blue hatched contours correspond to microlensing constraints from the EROS (Tisserand et al. 2007) and MACHO (Alcock et al. 2001) experiments, respectively. The green hatched curve corresponds to constraints from the stability of wide binary stars (Quinn et al. 2009). Note that the MACHO limits from these experiments extend to much lower and higher masses than are displayed in this plot.

that underwent multiple vigorous episodes of star formation. Crnojević et al. (2016) show that Eri II is possibly the least massive dwarf galaxy that is known to have an extended star formation history, and therefore its density profile may also be affected by baryons. The star cluster of Eri II may offer potential to constrain the dark matter profile of Eri II through survivability arguments (see, e.g., Cole et al. 2012) and could provide an independent probe of the dark matter profile shape. A better understanding of the dark matter distribution at small scales will help us understand how the dwarf galaxies we observe today are linked to the primordial population of dark matter subhalos predicted by $\Lambda \mathrm{CDM}$ cosmology.

\section{Summary}

We obtained Magellan/IMACS spectroscopy of stars in the recently discovered Milky Way satellite Eridanus II. We identified 28 members based on the radial velocities of 54 stars in the vicinity of Eri II. Of the confirmed members, the $\mathrm{S} / \mathrm{N}$ of 16 stars is high enough to measure their metallicity.

We find a systemic velocity of $v_{\text {hel }}=75.6 \pm 1.3$ (stat.) \pm 2.0 (sys.) $\mathrm{km} \mathrm{s}^{-1}\left(v_{\mathrm{GSR}}=-66.6 \mathrm{~km} \mathrm{~s}^{-1}\right)$ and a velocity dispersion of $6.9_{-0.9}^{+1.2} \mathrm{~km} \mathrm{~s}^{-1}$. The mass within the half-light radius of Eri II is $M_{1 / 2}=1.2_{-0.3}^{+0.4} \times 10^{7} M_{\odot}$, corresponding to a dynamical mass-to-light ratio of $420_{-140}^{+210} M_{\odot} / L_{\odot}$. The mean metallicity of Eri II is $[\mathrm{Fe} / \mathrm{H}]=-2.38 \pm 0.13$, with a metallicity dispersion of $\sigma_{[\mathrm{Fe} / \mathrm{H}]}=0.47_{-0.09}^{+0.12}$. The dynamical and chemical evidence both show that Eri II is a dark matter-dominated dwarf galaxy. 
The negative velocity of Eri II in the Galactic standard of rest frame implies that it is moving toward the Milky Way. Eri II is therefore either falling into the Milky Way potential for the first time, or it has recently passed the apocenter of its orbit. By identifying subhalos in dark matter-only simulations that are consistent with the line-of-sight velocity and distance of Eri II, we showed that Eri II is very likely bound to the Milky Way and is mostly likely on its second infall with an eccentric orbit. Future measurements of its proper motion will better constrain its orbit and confirm its origin. Furthermore, our measurements of radial velocities show that none of the candidate blue-loop stars we observed are associated with Eri II. We therefore conclude that there is no evidence of recent star formation ( $\sim 250 \mathrm{Myr})$ in Eri II.

Although Eri II is not a promising target for indirect searches for WIMP annihilation because of its distance, it offers a unique opportunity to constrain MACHO dark matter because it has a central star cluster. Our spectroscopic analysis provides a direct measurement of the mass and density of the Eri II dark matter halo and thus more precise constraints on the abundance of MACHO dark matter. Moreover, the existence of the star cluster may also offer an independent probe to constrain the dark matter density profile.

This paper has gone through internal review by the DES collaboration. We thank the anonymous referee for suggestions that substantially improved the quality of the paper. T.S.L. and J.D.S. thank Dan Kelson for many helpful conversations regarding IMACS data reduction. T.S.L. acknowledges support by the Mitchell Institute Fellowship from Texas A\&M University and the Leon Lederman Fellowship from Fermilab. J.D.S. acknowledges support from the National Science Foundation under grant AST-1108811. J.G.B. acknowledges support from Centro de Excelencia Severo Ochoa SEV-20120234 and SEV-2012-0249. E.B. acknowledges financial support from the European Research Council (ERC-StG335936).

This research has made use of NASA's Astrophysics Data System Bibliographic Services. This research made use of Astropy, a community-developed core Python package for Astronomy (Astropy Collaboration et al. 2013). Contour plots were generated using corner. py (Foreman-Mackey 2016).

Funding for the DES Projects has been provided by the U.S. Department of Energy, the U.S. National Science Foundation, the Ministry of Science and Education of Spain, the Science and Technology Facilities Council of the United Kingdom, the Higher Education Funding Council for England, the National Center for Supercomputing Applications at the University of Illinois at Urbana-Champaign, the Kavli Institute of Cosmological Physics at the University of Chicago, the Center for Cosmology and Astro-Particle Physics at the Ohio State University, the Mitchell Institute for Fundamental Physics and Astronomy at Texas A\&M University, Financiadora de Estudos e Projetos, Fundação Carlos Chagas Filho de Amparo à Pesquisa do Estado do Rio de Janeiro, Conselho Nacional de Desenvolvimento Científico e Tecnológico and the Ministério da Ciência, Tecnologia e Inovação, the Deutsche Forschungsgemeinschaft and the Collaborating Institutions in the Dark Energy Survey.

The Collaborating Institutions are Argonne National Laboratory, the University of California at Santa Cruz, the University of Cambridge, Centro de Investigaciones Energéticas,
Medioambientales y Tecnológicas-Madrid, the University of Chicago, University College London, the DES-Brazil Consortium, the University of Edinburgh, the Eidgenössische Technische Hochschule (ETH) Zürich, Fermi National Accelerator Laboratory, the University of Illinois at UrbanaChampaign, the Institut de Ciències de l'Espai (IEEC/CSIC), the Institut de Física d'Altes Energies, Lawrence Berkeley National Laboratory, the Ludwig-Maximilians Universität München and the associated Excellence Cluster Universe, the University of Michigan, the National Optical Astronomy Observatory, the University of Nottingham, The Ohio State University, the University of Pennsylvania, the University of Portsmouth, SLAC National Accelerator Laboratory, Stanford University, the University of Sussex, Texas A\&M University, and the OzDES Membership Consortium.

The DES data management system is supported by the National Science Foundation under Grant Number AST1138766. The DES participants from Spanish institutions are partially supported by MINECO under grants AYA201239559, ESP2013-48274, FPA2013-47986, and Centro de Excelencia Severo Ochoa SEV-2012-0234 and SEV-20120249, some of which include ERDF funds from the European Union. Research leading to these results has received funding from the European Research Council under the European Unions Seventh Framework Programme (FP7/2007-2013) including ERC grant agreements 240672, 291329, and 306478.

Facility: This paper includes data gathered with the 6.5 meter Magellan Telescopes located at Las Campanas Observatory, Chile.

\section{References}

Abbott, B. P., Abbott, R., Abbott, T. D., et al. 2016, PhRvL, 116, 061102 Adams, J. J., Simon, J. D., Fabricius, M. H., et al. 2014, ApJ, 789, 63 Alcock, C., Allsman, R. A., Alves, D. R., et al. 2001, ApJL, 550, L169 Astropy Collaboration, Robitaille, T. P., Tollerud, E. J., et al. 2013, A\&A, 558, A33

Baltz, E., Berenji, B., Bertone, G., et al. 2008, JCAP, 0807, 013

Barnes, D. G., Staveley-Smith, L., de Blok, W. J. G., et al. 2001, MNRAS, 322,486

Bechtol, K., Drlica-Wagner, A., Balbinot, E., et al. 2015, ApJ, 807, 50

Bergström, L., \& Snellman, H. 1988, PhRvD, 37, 3737

Bird, S., Cholis, I., Muñoz, J. B., et al. 2016, PhRvL, 116, 201301

Bland-Hawthorn, J., \& Gerhard, O. 2016, ARA\&A, 54, 529

Blitz, L., \& Robishaw, T. 2000, ApJ, 541, 675

Bovy, J., Allende Prieto, C., Beers, T. C., et al. 2012, ApJ, 759, 131

Brandt, T. D. 2016, ApJL, 824, L31

Bressan, A., Marigo, P., Girardi, L., et al. 2012, MNRAS, 427, 127

Brown, T. M., Tumlinson, J., Geha, M., et al. 2014, ApJ, 796, 91

Brüns, C., Kerp, J., Staveley-Smith, L., et al. 2005, A\&A, 432, 45

Bullock, J. S., Kravtsov, A. V., \& Weinberg, D. H. 2001, ApJ, 548, 33

Carrera, R., Pancino, E., Gallart, C., \& del Pino, A. 2013, MNRAS, 434, 1681

Charbonnier, A., Combet, C., Daniel, M., et al. 2011, MNRAS, 418, 1526

Clesse, S., \& García-Bellido, J. 2015, PhRvD, 92, 023524

Clesse, S., \& García-Bellido, J. 2016, PDU, 10, 002

Cole, D. R., Dehnen, W., Read, J. I., \& Wilkinson, M. I. 2012, MNRAS, 426, 601

Collins, M. L. M., Tollerud, E. J., Sand, D. J., et al. 2016, MNRAS, 467, 573

Cooper, M. C., Newman, J. A., Davis, M., Finkbeiner, D. P., \& Gerke, B. F. 2012, spec2d: DEEP2 DEIMOS Spectral Pipeline, Astrophysics Source Code Library, ascl:1203.003

Crnojević, D., Sand, D. J., Zaritsky, D., et al. 2016, ApJL, 824, L14

de Blok, W. J. G., McGaugh, S. S., Bosma, A., \& Rubin, V. C. 2001, ApJL, $552, \mathrm{~L} 23$

de Jong, J. T. A., Harris, J., Coleman, M. G., et al. 2008, ApJ, 680, 1112 Di Cintio, A., Brook, C. B., Macciò, A. V., et al. 2014, MNRAS, 437, 415 Dressler, A., Bigelow, B., Hare, T., et al. 2011, PASP, 123, 288

Dressler, A., Hare, T., Bigelow, B. C., \& Osip, D. J. 2006, Proc. SPIE, 6269, $62690 \mathrm{~F}$

Drlica-Wagner, A., Albert, A., Bechtol, K., et al. 2015a, ApJL, 809, L4 
Drlica-Wagner, A., Bechtol, K., Allam, S., et al. 2016, ApJL, 833, L5

Drlica-Wagner, A., Bechtol, K., Rykoff, E. S., et al. 2015b, ApJ, 813, 109

Dubinski, J., \& Carlberg, R. G. 1991, ApJ, 378, 496

Einasto, J., Saar, E., Kaasik, A., \& Chernin, A. D. 1974, Natur, 252, 111

Essig, R., Sehgal, N., \& Strigari, L. E. 2009, PhRvD, 80, 023506

Fillingham, S. P., Cooper, M. C., Pace, A. B., et al. 2016, MNRAS, 463, 1916

Fillingham, S. P., Cooper, M. C., Wheeler, C., et al. 2015, MNRAS, 454, 2039

Foreman-Mackey, D. 2016, J. Open Source Softw., 24

Foreman-Mackey, D., Hogg, D. W., Lang, D., \& Goodman, J. 2013, PASP, 125,306

Garrison-Kimmel, S., Boylan-Kolchin, M., Bullock, J. S., \& Lee, K. 2014, MNRAS, 438, 2578

Geringer-Sameth, A., Koushiappas, S. M., \& Walker, M. 2015, ApJ, 801, 74

Governato, F., Zolotov, A., Pontzen, A., et al. 2012, MNRAS, 422, 1231

Grcevich, J., \& Putman, M. E. 2009, ApJ, 696, 385

Gunn, J. E., Lee, B. W., Lerche, I., Schramm, D. N., \& Steigman, G. 1978, ApJ, 223, 1015

Homma, D., Chiba, M., Okamoto, S., et al. 2016, ApJ, 832, 21

Irwin, M. J., Belokurov, V., Evans, N. W., et al. 2007, ApJL, 656, L13

Kim, D., \& Jerjen, H. 2015, ApJL, 808, L39

Kim, D., Jerjen, H., Mackey, D., Da Costa, G. S., \& Milone, A. P. 2015a, ApJL, 804, L44

Kim, D., Jerjen, H., Milone, A. P., Mackey, D., \& Da Costa, G. S. 2015b, ApJ, 803,63

Kirby, E. N., Cohen, J. G., Guhathakurta, P., et al. 2013, ApJ, 779, 102

Komatsu, E., Smith, K. M., Dunkley, J., et al. 2011, ApJS, 192, 18

Koposov, S. E., Belokurov, V., Torrealba, G., \& Evans, N. W. 2015, ApJ, 805,130

Kruijssen, J. M. D., \& Lützgendorf, N. 2013, MNRAS, 434, L41

Laevens, B. P. M., Martin, N. F., Bernard, E. J., et al. 2015a, ApJ, 813, 44

Laevens, B. P. M., Martin, N. F., Ibata, R. A., et al. 2015b, ApJL, 802, L18

Martin, N. F., \& Jin, S. 2010, ApJ, 721, 1333

Martin, N. F., Nidever, D. L., Besla, G., et al. 2015, ApJL, 804, L5

Martinez, G. D. 2015, MNRAS, 451, 2524

McConnachie, A. W. 2012, AJ, 144, 4

McConnachie, A. W., \& Côté, P. 2010, ApJL, 722, L209

Minor, Q. E., Martinez, G., Bullock, J., Kaplinghat, M., \& Trainor, R. 2010, ApJ, 721, 1142
Navarro, J. F., Frenk, C. S., \& White, S. D. M. 1996, ApJ, 462, 563

Olszewski, E. W., Pryor, C., \& Armandroff, T. E. 1996, AJ, 111, 750

Pontzen, A., \& Governato, F. 2012, MNRAS, 421, 3464

Quinn, D. P., Wilkinson, M. I., Irwin, M. J., et al. 2009, MNRAS, 396, L11

Ricotti, M. 2009, MNRAS, 392, L45

Robin, A. C., Reylé, C., Derrière, S., \& Picaud, S. 2003, A\&A, 409, 523

Ryan-Weber, E. V., Begum, A., Oosterloo, T., et al. 2008, MNRAS, 384, 535

Schiavon, R. P., Barbuy, B., Rossi, S. C. F., \& Milone, A. 1997, ApJ, 479, 902

Schönrich, R., Binney, J., \& Dehnen, W. 2010, MNRAS, 403, 1829

Simon, J. D., Drlica-Wagner, A., Li, T. S., et al. 2015, ApJ, 808, 95

Simon, J. D., \& Geha, M. 2007, ApJ, 670, 313

Simon, J. D., Geha, M., Minor, Q. E., et al. 2011, ApJ, 733, 46

Simon, J. D., Li, T. S., Drlica-Wagner, A., et al. 2016, arXiv:1610.05301

Sohn, S. T., Majewski, S. R., Muñoz, R. R., et al. 2007, ApJ, 663, 960

Spekkens, K., Urbancic, N., Mason, B. S., Willman, B., \& Aguirre, J. E. 2014 ApJL, 795, L5

Stanimirović, S., Staveley-Smith, L., \& Jones, P. A. 2004, ApJ, 604, 176

Strigari, L. E., Koushiappas, S. M., Bullock, J. S., et al. 2008, ApJ, 678, 614

Taylor, C., Boylan-Kolchin, M., Torrey, P., Vogelsberger, M., \& Hernquist, L. 2016, MNRAS, 461, 3483

Tisserand, P., Le Guillou, L., Afonso, C., et al. 2007, A\&A, 469, 387

Torrealba, G., Koposov, S. E., Belokurov, V., \& Irwin, M. 2016a, MNRAS, 459, 2370

Torrealba, G., Koposov, S. E., Belokurov, V., et al. 2016b, MNRAS, 463, 712

van der Marel, R. P., Alves, D. R., Hardy, E., \& Suntzeff, N. B. 2002, AJ, 124,2639

Walker, M. G., Mateo, M., Olszewski, E. W., et al. 2006, AJ, 131, 2114

Walker, M. G., \& Penarrubia, J. 2011, ApJ, 742, 20

Weisz, D. R., Dolphin, A. E., Skillman, E. D., et al. 2014, ApJ, 789, 147

Weisz, D. R., Zucker, D. B., Dolphin, A. E., et al. 2012, ApJ, 748, 88

Westmeier, T., Staveley-Smith, L., Calabretta, M., et al. 2015, MNRAS, 453, 338

Wetzel, A. R., Tollerud, E. J., \& Weisz, D. R. 2015, ApJL, 808, L27

Wheeler, C., Oñorbe, J., Bullock, J. S., et al. 2015, MNRAS, 453, 1305

Wolf, J., Martinez, G. D., Bullock, J. S., et al. 2010, MNRAS, 406, 1220 